\title{
Fluorescent Carbon Nanoparticles for Sensitive and Selective Detection of Palladium (Pd2+)
}

Sichen Zhang

sichen.zhang@uconn.edu

\section{Recommended Citation}

Zhang, Sichen, "Fluorescent Carbon Nanoparticles for Sensitive and Selective Detection of Palladium (Pd2+)" (2016). Master's Theses. 929.

https://opencommons.uconn.edu/gs_theses/929

This work is brought to you for free and open access by the University of Connecticut Graduate School at OpenCommons@UConn. It has been accepted for inclusion in Master's Theses by an authorized administrator of OpenCommons@UConn. For more information, please contact opencommons@uconn.edu. 
Fluorescent Carbon Nanoparticles for Sensitive and Selective Detection of Palladium $\left(\mathbf{P d}^{2+}\right)$

\author{
Sichen Zhang \\ B.S., Hefei University, 2011
}

A Thesis Submitted in partial Fulfillment of the

Requirement for the degree of

Master of Science

At the

University of Connecticut

2016 


\section{Copyright by}

Sichen Zhang

2016 


\section{APPROVAL PAGE}

Masters of Science Thesis

Fluorescent Carbon Nanoparticles for Sensitive and Selective Detection of Palladium $\left(\mathrm{Pd}^{2+}\right)$

Presented by

Sichen Zhang, B.S.

Major Advisor

Yu Lei

Associate Advisor

\section{Christian Bruckner}

Associate Advisor

Guoan Zheng

University of Connecticut

2016 


\section{Acknowledgement}

I would like to express my deepest appreciation to my major advisor, Professor Yu Lei, for his guidance and continuous support in my master's period. I have gained a lot of experience on research and life under his supervision.

Furthermore, I would like to thank my co-advisors, Professor Christian Bruckner and Professor Zheng Guoan. They have provided constructive suggestions and insightful advices to help me to complete my program.

I want to take this opportunity to thank Professor Hong Chen, who granted me the knowledge of organic chemistry and initiated my interest for further education.

In the two past years, my dealing labmates, Dr. Xiangcheng Sun, Jun Chen, Xiaoyu Ma, Qiuchen Dong, Rafaela de Silverira and Dongwook Kawa have shared their experience on research and helped me a lot in the daily life.

I also would like to appreciate Professor Jie He and Junkai He for their assistance for the characterization techniques and analysis.

Finally, I would like to thank my parents and my friends. Without their unlimited love and support, I cannot realize my dream for master degree. I also want to thank Yang for his help and companionship. 


\section{Contents}

APPROVAL PAGE

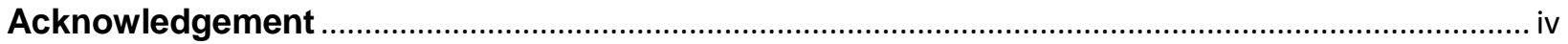

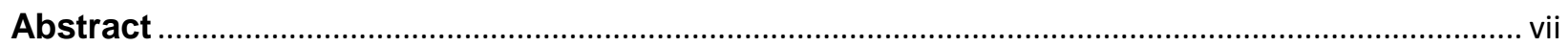

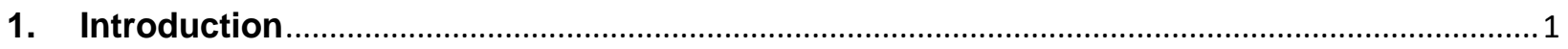

1.1. Fluorescent carbon nanoparticles and their preparation methods and applications ....... 1

1.1.1. Fluorescent carbon nanoparticles...................................................................... 1

1.1.2. Preparation methods of carbon nanoparticles ............................................................ 2

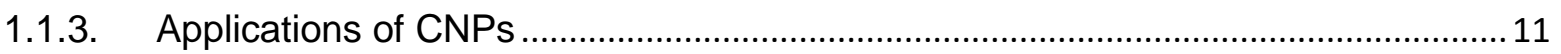

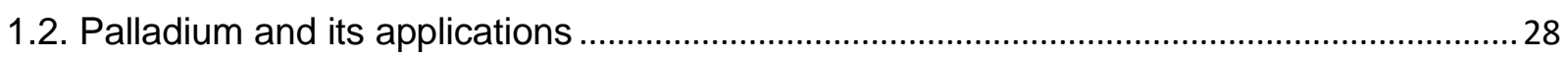

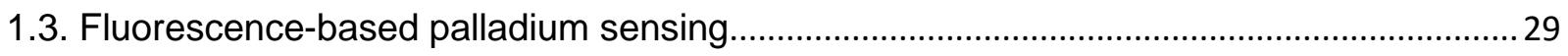

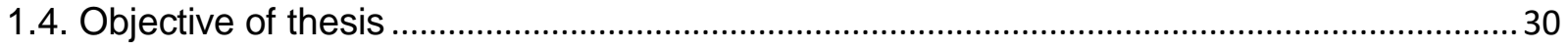

2. DESIGN OF CNPS-BASED FLUORESCENCE PROBE FOR PALLADIUM SENSING.................31

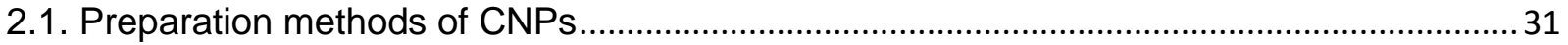

2.2. The design of CNPs-based fluorescence chemosensor for $\mathrm{Pd}^{2+}$ sensing......................... 31

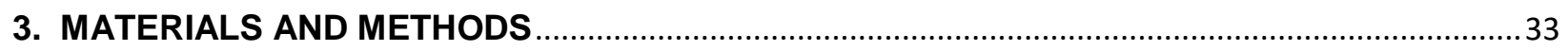

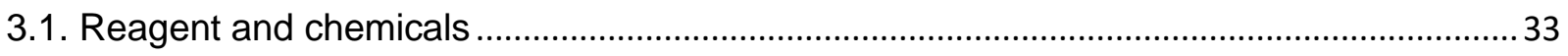

3.2. Preparation of fluorescent carbon nanoparticles ............................................................ 33

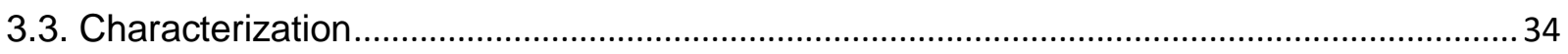

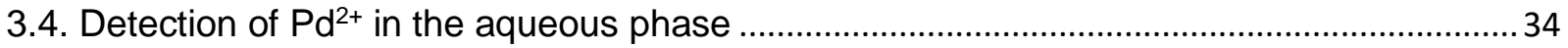

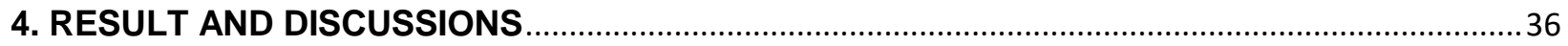

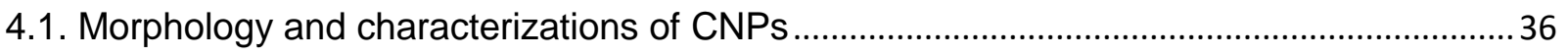

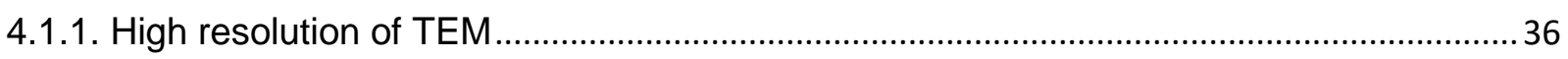

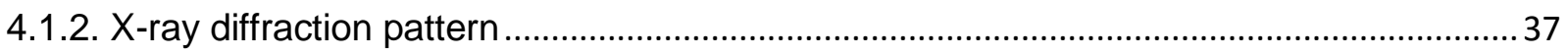

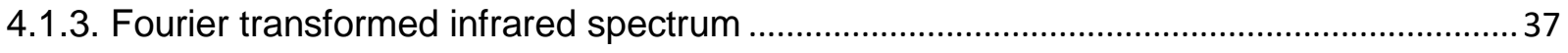

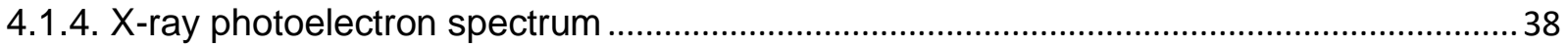

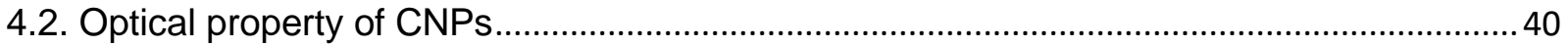

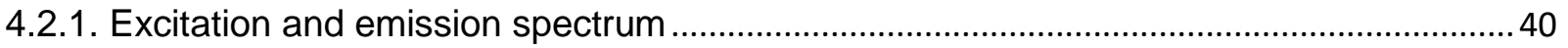

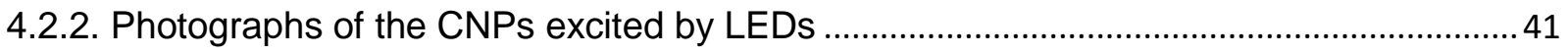

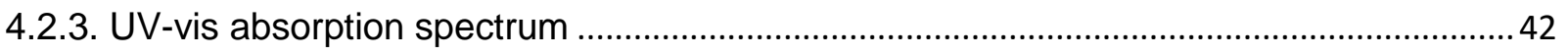

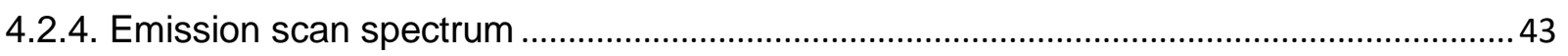

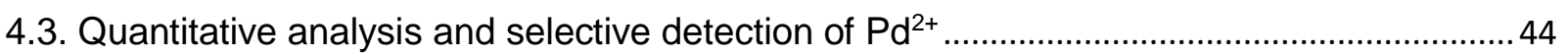




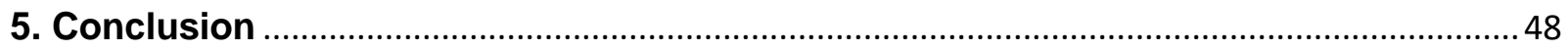

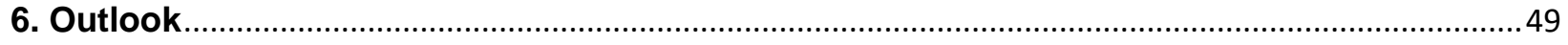

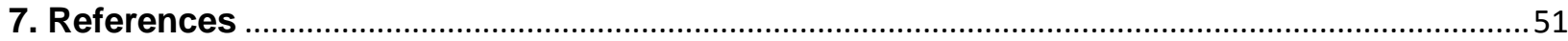




\begin{abstract}
Fluorescent carbon nanoparticles (CNPs) was synthesized using well-known citric acid and polyethylenimine precursors but under a modified microwave reaction conditions in order to achieve high sensitivity and selectivity for $\mathrm{Pd}^{2+}$. The assynthesized fluorescent CNPs with an average diameter of 2-3 nm exhibited good water solubility and photo-stability. The CNPs were systematically characterized using various advanced techniques and the fluorescence study on the as-prepared CNPs showed the excitation-dependent emission properties. Finally, the as-synthesized carbon nanoparticles served as a promising fluorescence probe for $\mathrm{Pd}^{2+}$ sensing. The sensitivity and the selectivity for $\mathrm{Pd}^{2+}$ detection were investigated by fluorescence quenching titrations and comparison of various competing metal ions, respectively. The results show that besides the excellent selectivity to $\mathrm{Pd}^{2+}$ against other metal ions, the limit of detection to $\mathrm{Pd}^{2+}$ could also reach as low as $12.4 \mathrm{nM}$, which is much lower than the threshold concentration limit of $\mathrm{Pd}^{2+}$ in medicine and environmental samples. The findings here indicate that the as-prepared CNPs hold great promise as a low-cost sensing material for sensitive and selective detection of palladium.
\end{abstract}

Keywords: Fluorescent carbon nanoparticles, Sensitivity, Selectivity, Limit of detection, Fluorescence probe. 


\section{Introduction}

1.1. Fluorescent carbon nanoparticles and their preparation methods and applications

\subsubsection{Fluorescent carbon nanoparticles}

Carbon nanomaterials including carbon nanotubes, graphene sheets, nanodiamonds, fullerenes and fluorescent carbon nanoparticles have attracted much research interest in recent years. Especially, carbon nanoparticles (CNPs), because of their excellent optical properties, biocompatibility, water solubility, photo-stability, and ease of cost-effective synthesis, have gained tremendous attention in a wide spectrum of chemical and biomedical applications, ranging from sensing to bioimaging, fluorescent ink and phototherapy. In recent years, fluorescent CNPs have been regarded as a promising candidate to replace the conventional semiconductor quantum dots in various applications ${ }^{1}$.

Fluorescent CNPs were coincidentally discovered and produced in the process of purification of single-wall carbon nanotubes derived from candle soot by $\mathrm{Xu}$ et al ${ }^{2}$. This finding of fluorescent CNPs initiated tremendous research interests on this type of novel nanoscale carbon materials around the world. However, CNPs typically suffer from low fluorescence quantum yield in the early development stage. With the improvement of synthetic methods and doping of different elements into CNPs, the fluorescent

photoluminescence $(\mathrm{PL})$ properties are greatly improved and thus suitable for a wide 
range of applications, such as bioimaging ${ }^{3}$, biosensing ${ }^{4,5}$, biomedicine ${ }^{6}$, heavy metal ions sensing 7,8 and explosive detection ${ }^{9,}{ }^{10}$, light harvesting ${ }^{11}$.

\subsubsection{Preparation methods of carbon nanoparticles}

Carbon dots can be prepared by a number of methods but that all generally fall into two broad categories: bottom-up and top-down approaches. In bottom-up approaches, the CNPs are obtained from assembling molecular precursors under a range of different reaction conditions, including hydrothermal, microwave-assisted, ultrasonic, acid dehydration, and pyrolytic condition. CNPs could be prepared through "Top down" approaches from large cluster of carbon materials, such as carbon nanotubes, graphite, carbon soot and so on through arc-charge, laser ablation and chemical oxidation. The preparation methods of CNPs will be briefly discussed in the subsequent sections.

\subsubsection{Hydrothermal method}

Hydrothermal method has been widely employed in the synthesis of nanostructured materials. Recently, Cui et al. ${ }^{12}$ reported the fabrication of $\mathrm{N}$-doped carbon dots using ammonium citrate, ammonium hydroxide and hydrogen peroxide as precursors under a hydrothermal condition of $180{ }^{\circ} \mathrm{C}$ for $2 \mathrm{~h}$. The as-prepared $\mathrm{N}$-doped CNPs showed strong green fluorescence with quantum yield of $15.7 \%$. During the synthesis, ammonium hydroxide served as nitrogen resource on one hand; on the other hand, the release of $\mathrm{NH}_{3}$ from ammonium hydroxide during the hydrothermal process resulted in high-pressured alkaline environment, which favors the decomposition of ammonium citrate to form of CNPs. The role of $\mathrm{H}_{2} \mathrm{O}_{2}$ in the synthesis is to shift the emission band of CNPs from blue to green emission due to its hydroxyl group. It was interesting to find 
that the effect of $\mathrm{H}_{2} \mathrm{O}_{2}$ in this hydrothermal method was different from that of previous reports in which $\mathrm{H}_{2} \mathrm{O}_{2}$ is usually employed as the fluorescence quencher ${ }^{13,14}$

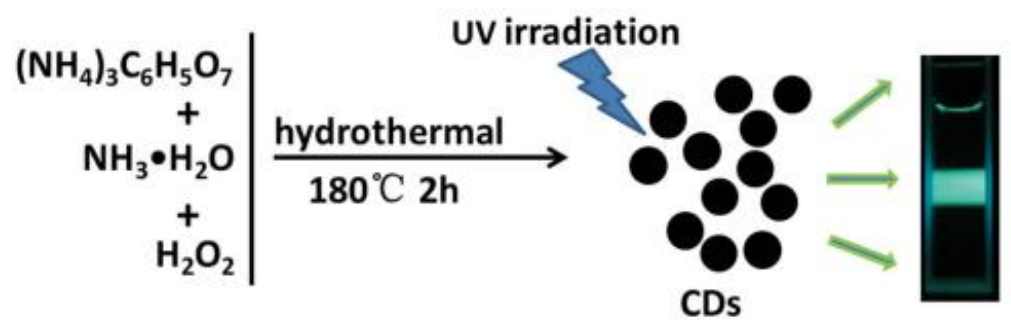

Figure 1. Hydrothermal synthesis of green fluorescent carbon nanoparticles using ammonium citrate, ammonium hydroxide and hydroxide peroxide as precursors.

\subsubsection{Microwave-assisted method}

Due to its simplicity, low cost, fast reaction rate and the ability to generate a variety of CNPs in a single step, microwave-assisted method has also become popular in the synthesis of fluorescent carbon nanoparticles with diverse optical properties. In general, microwave heating of the precursor solution resulted in the high temperature and/or high pressure environment to trigger the decomposition/reaction of precursors, thus forming CNPs. Recently, Qu and co-workers ${ }^{15}$ synthesized the dual emission fluorescent carbon nanoparticles, which simply used citric acid and urea as precursors. Under microwave-assisted conditions of 4-5 mins in a domestic $750 \mathrm{~W}$ microwave, the CNPs composed of $\mathrm{C}, \mathrm{H}, \mathrm{N}$ and $\mathrm{O}$ and hydroxyl, carboxyl and amine groups, which endowed the as-prepared CNPs with high water solubility, photostability and unique fluorescence property, were synthesized. However, the use of multiple precursors endows the difficulty to understand the synthetic mechanism. Very recently, our group synthesized blue fluorescent carbon dots using a single precursor (ammonium citrate dibasic) and a home-use microwave oven in less than one minute. ${ }^{16}$ It is believed that the fluorescent carbon nanoparticles are formed due to polymerization of the precursor 
ammonium citrate dibasic followed by partial carbonization of the resulting polymer. It is presumably assumed that ammonium citrate dibasic is condensed and inter-molecularly dehydrated to form oligomers when the reaction temperature is relatively low. Further increasing the temperature due to microwave heating produces a burst of high concentration of nucleation, which grows uniformly and is further carbonized to form noncrystalline carbon nanoparticles with hydrophilic functional groups on the surface. Both the ammonium and citrate groups in ammonium citrate dibasic contribute to the formation of fluorescent carbon nanoparticles. Control experiments under similar conditions but using citric acid or ammonium carbonate respectively were conducted, and after microwave heating we observed little fluorescence under UV lamp. Following this study, the same group also synthesized N,P co-doped CDs with an average diameter of $8.1 \mathrm{~nm}$ (Figure 2) and dual fluorescence emission (Figure 3) and ultrahigh quantum yield through microwave-assisted approach ${ }^{~}$.

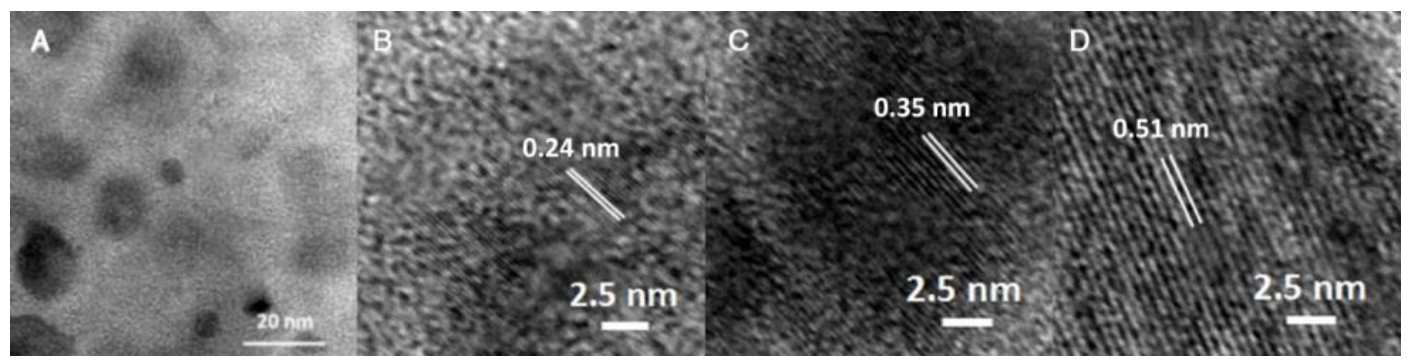

Figure 2. (A) TEM graph of the N,P-doped CNPs; (B-D) HRTEM images and lattice structures of different N,P-doped CNPs. 

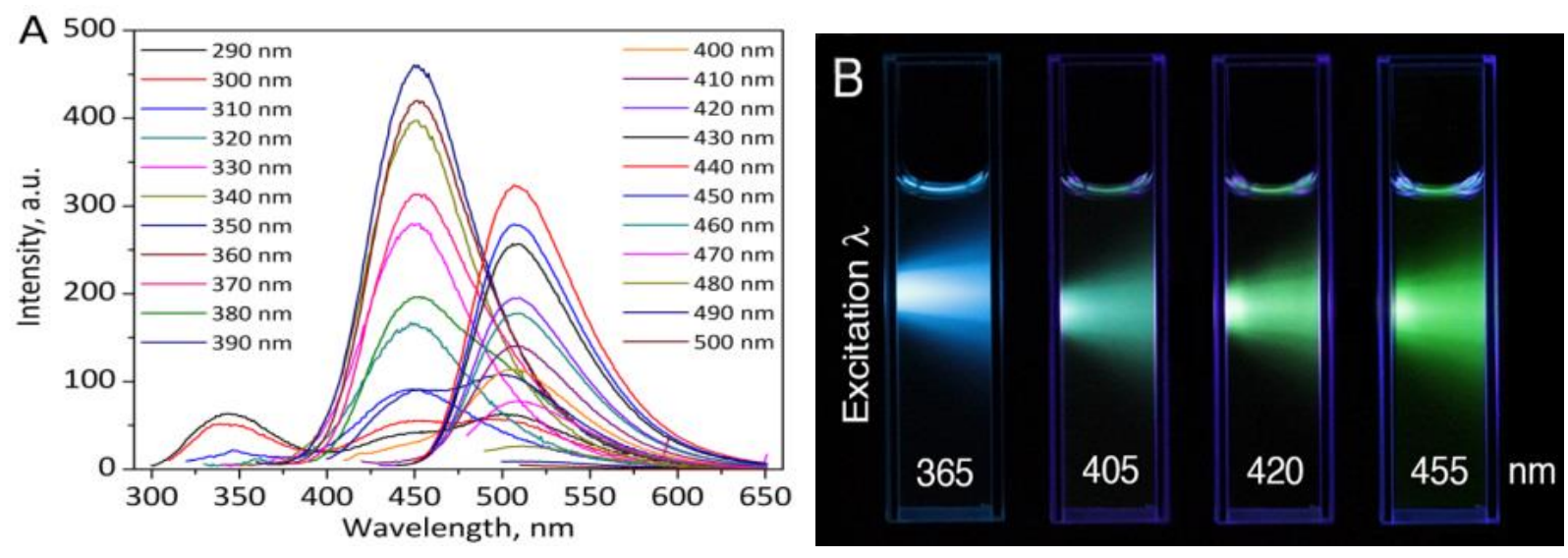

Figure 3. (A) Emission spectra (in water) of N,P-CDs at the various excitation wavelengths indicated. (B) Photographs of diluted N,P-CDs solutions (in water; quartz cuvettes) excited with fibreoptics-coupled LEDs of the wavelengths indicated, showing their excitation dependent emission properties.

\subsubsection{Pyrolytic method}

Pan et al. ${ }^{17}$ and Zhou et al. ${ }^{18}$ developed a pyrolytic method to prepare CNPs. In brief, EDTA-2Na-2 $\mathrm{H}_{2} \mathrm{O}$ was used as the precursor and filled in a quartz boat, and then thrusted into a quartz tube which was calcined at $400^{\circ} \mathrm{C}$ for 2 hours under nitrogen environment. Because of the incomplete pyrolysis of the precursor, $\mathrm{H}, \mathrm{N}, \mathrm{O}$ and $\mathrm{Na}$ are incorporated into the CNPs. The obtained black powder was then dissolved in acetone and centrifuged for 15 mins to remove the unreacted compounds. TEM characterization shows that the CNPs were spherical with the mean size of $3.8 \mathrm{~nm}$, while the FTIR spectra in Figure 4 reveals that the peaks at around $1470 \mathrm{~cm}^{-1}$ and $1647 \mathrm{~cm}^{-1}$ were assigned to carboxylic group, and the peaks appeared at $3346 \mathrm{~cm}^{-1}$ was attributed to the presence of $\mathrm{O}-\mathrm{H}$ bond vibration. The surface groups not only promoted hydrophilicity of the CNPs, but also enhanced their PL property. 


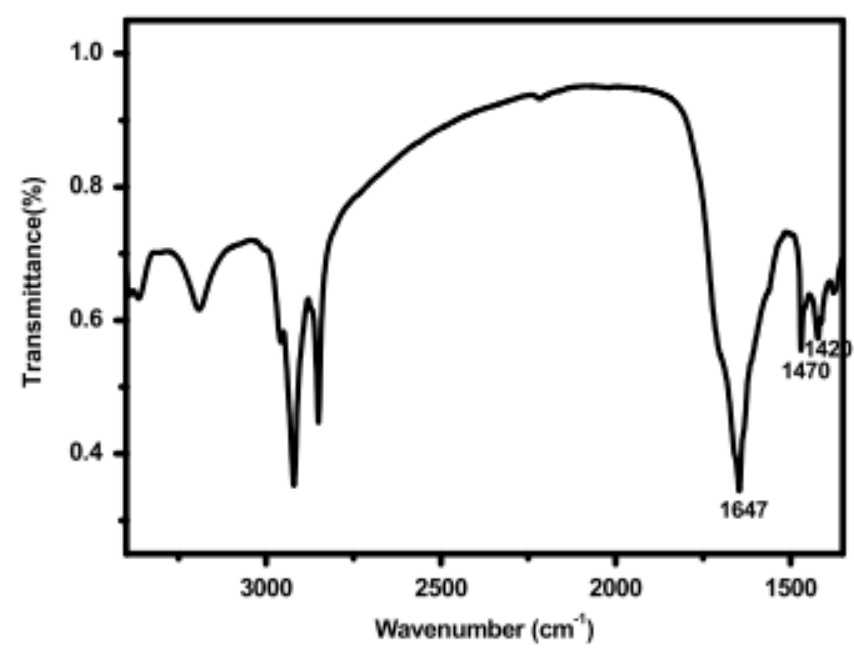

Figure 4. Fourier transform infrared (FTIR) image of CNPs for functional group analysis.

\subsubsection{Acid dehydration method}

Citric acid, phosphate, sulfuric acid and nitrate have already been employed for the preparation of fluorescent carbon nanoparticles. Wang et al. ${ }^{11}$ reported the synthesis of nitrogen-rich fluorescent CNPs utilizing melamine and glycerol as precursors, and citric acid, $\mathrm{H}_{2} \mathrm{SO}_{4}$ or $\mathrm{H}-\mathrm{ZSM}-5$ as acid catalyst (Figure 5). Specifically, in this acid dehydration method, melamine was dissolved in glycerol and subsequently heated up to $270{ }^{\circ} \mathrm{C}$ with nitrogen flowing. Citric acid was quickly added into the high temperature solution and maintained at $270{ }^{\circ} \mathrm{C}$ for 15 mins and then cooled down to room temperature to obtain the dark brown solution of CNPs. Following by centrifugation and dialysis procedure, pure CNPs solution was obtained. Three fractions of CNPs with different diameters were obtained by using two dialysis membranes with molecular weight cut-off (MWCO) 3500 and MWCO 1000. The higher oxygen content accompanied with lower carbon percentage for small diameter fraction of CNPs suggests that the fraction of CNPs with larger diameter has undergone further dehydration and condensation. According to the FTIR spectra, the appearance of new surface functional groups and similar surface 
structure of the CNPs indicated the CNPs were produced through one spot reaction between melamine and glycerol. The effect of citric acid serving as catalyst was also investigated. The quantum yield of the CNPs obtained without the use of citric acid was about $50 \%$ lower than those underwent acid dehydration reaction.
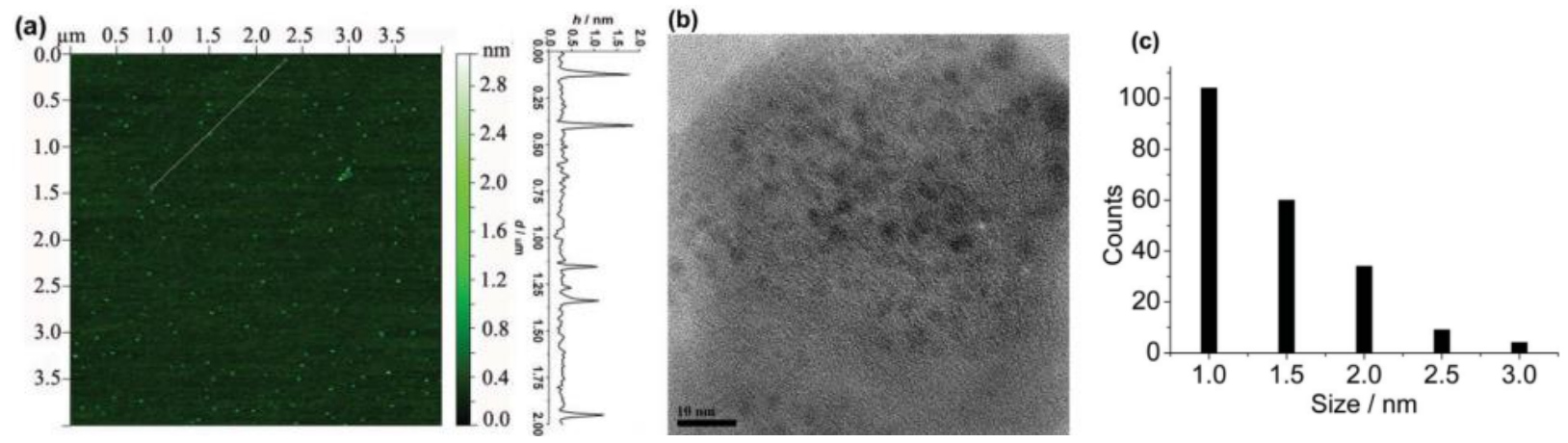

Figure 5, AFM (a) and TEM (b) images of CNPs, and the size distribution of CNPs (c) based on AFM image analysis.

\subsubsection{Arc-discharge method}

Fluorescent carbon nanoparticles were firstly reported by Xu's group ${ }^{2}$ when they purified single-walled carbon nanotubes (SWNTs) from arc-discharge soot. Three discriminate substances, which were long nanotubes, tubular material and fluorescent carbon material (Figure 6), were obtained in the process of purification of arc-discharge soot suspension through electrophoretic method. The separated and purified fluorescent carbon materials were further segregated based on the different color of fluorescence emission under $366 \mathrm{~nm}$ excitation. The molecule weight of the fluorescent carbon materials could be correlated to the emission wavelength of fluorescent materials, and the nominal molecular weights of the green-blue, yellow and orange were $3000-10000,10000-30000,30000-50000$, respectively. Carboxylic groups were functionalized on the as-prepared carbon nanomaterials which were proved by FTIR 
characterization. In conjunction with electrophoresis-based separation, arc-discharge method offers a facile way to prepare CNPs.

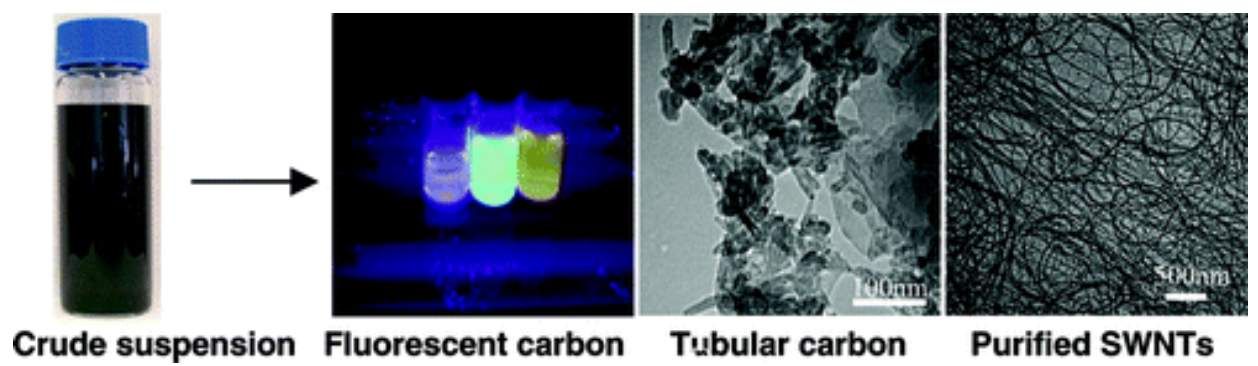

Figure 6. Fluorescent CNPs, tubular carbon and purified SWNTs prepared through arc-discharge method. Reference with permission.

\subsubsection{Laser ablation}

Adapting a top-down approach, Hu et al. ${ }^{19}$ reported a novel one-step synthesis of fluorescent carbon nanoparticles, utilizing laser irradiation of carbon powders in organic solvent such as diamine hydrate, diethanolamine, and poly(ethylene glycol) (PEG200N). Taking PEG $200 \mathrm{~N}$ as an example of a representative solvent (Figure 7), graphite powders were dispersed in $\mathrm{PEG}_{200 \mathrm{~N}}$ followed by simultaneous treatment of laser irradiation and ultrasonication for 2 hours. Laser irradiation was conducted with a wavelength of 1.064 $\mu \mathrm{m}$ and power density of $6.0 \times 10^{6} \mathrm{~W} \mathrm{~cm}^{-2}$ while ultrasound was employed to facilitate the movement of carbon particles. The black supernatant (Sample A) was obtained from the suspension via centrifugation. In order to understand the relationship between surface functional groups and PL of fluorescent carbon nanoparticles, both the suspension of graphite powders in DI water using the same procedure described above and the suspension of graphite powders in DI water followed by subsequently heating in the mixture of perchloric acid $\left(\mathrm{HClO}_{4}\right)$ and $\mathrm{PEG}_{200 \mathrm{~N}}$ (Sample B) were used as a control group (Figure 8). The CNPs obtained from the supernatant of graphite powders 
dispersed in $\mathrm{PEG}_{200 \mathrm{~N}}$ and the mixture of $\mathrm{HClO}_{4}$ and $\mathrm{PEG}_{200 \mathrm{~N}}$ with graphite powder had emission peak of $490 \mathrm{~nm}$, while that synthesized in DI water did not show any PL emission. In addition, the functional group such as carboxylate group appeared on the fluorescent CNPs, but was not presented in non-fluorescent materials. These results not only indicated the effect of the solvent used on the synthesis of fluorescent CNPs but also revealed that the surface functional groups played an important role in the origin of the fluorescence. Further study of the effect of the carbon source and organic solvent on the fluorescence properties of carbon dots concluded that the PL property of CNPs was not obviously influenced by the type of carbon source in the same organic solvent. However, the emission peaks of the CNPs obtained in different solvents $\left(\mathrm{PEG}_{200 \mathrm{~N}}\right.$, diamine hydrate and diethanolamine) were different due to the formation of different surface functional groups. Furthermore, the PL peak of CNPs shifted to a longer wavelength with increasing of PEG molecular weights was also proposed.

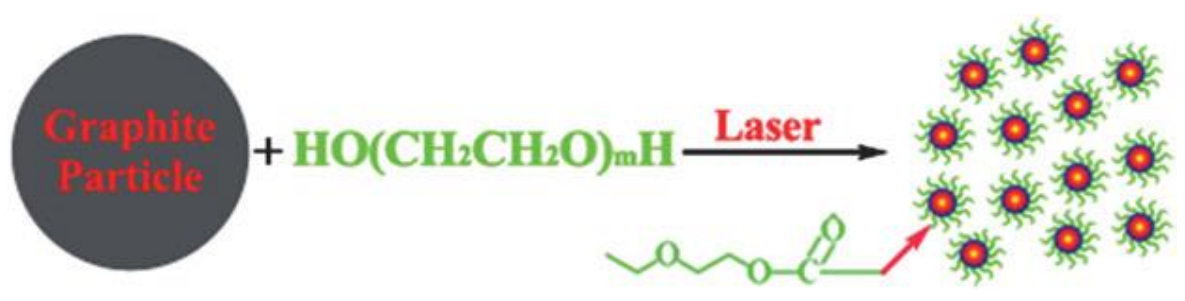

Figure 7. Schematic map of the one-step synthesis of luminescent CNPs in PEG 200 s solvent. 

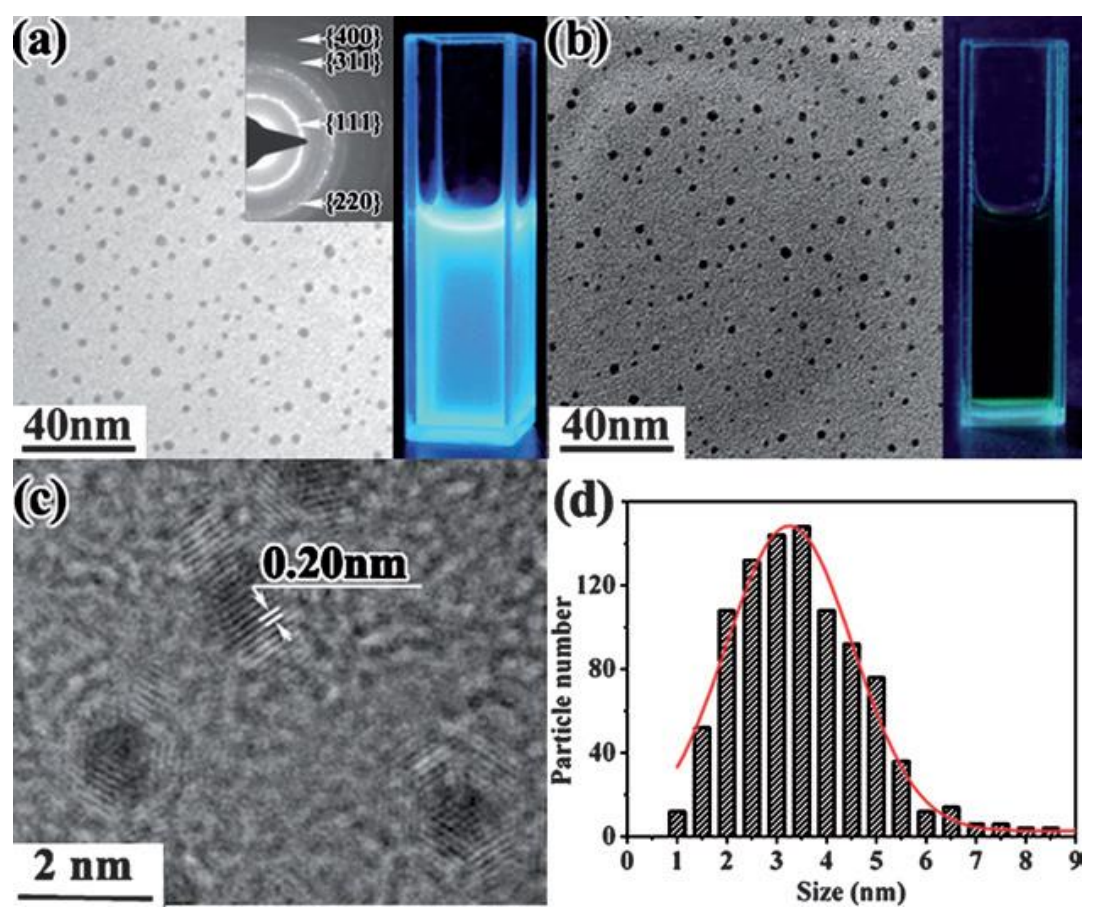

Figure 8. Representative TEM images of the CNPs and direct photographs of suspensions under a $365 \mathrm{~nm}$ UV lamp. (a) TEM image of the CNPs in sample A and direct photograph of sample A under a $365 \mathrm{~nm}$ UV lamp, the inset is the SAED pattern of the CNPs; (b) TEM image of the CNPs in sample B and direct photograph of sample B under a $365 \mathrm{~nm}$ UV lamp; (c) HRTEM image of the CNPs in sample A; (d) the size distribution of the CNPs in sample $A$.

\subsubsection{Chemical oxidation}

Utilizing candle soot, a common carbon source, with an oxidative acid treatment, Liu et al. ${ }^{20}$ prepared multi-color fluorescent CNPs (Figure 9). The candle soot was treated with $5 \mathrm{M} \mathrm{HNO}_{3}$ and refluxed for 12 hours, and then the supernatant was separated from the suspension by centrifugation. The pure fluorescent CNPs were separated from the neutralized candle-soot dispersion through denaturing PAGE gel electrophoresis. The fluorescent carbon nanoparticles separated in the PAGE gel showed distinguished colorful bands under $321 \mathrm{~nm}$ UV light. The different CNPs were collected from the gel and dissolved in DI water. The PL spectra showed that the emission peaks of different CNPs were ranged from $415 \mathrm{~nm}$ to $615 \mathrm{~nm}$ under the same UV light. However, the quantum yields of the as-synthesized CNPs were lower than $1 \%$, relatively poorer than 
other literature reports. In addition, the PL properties of the CNPs shows strong $\mathrm{pH}$ dependency, and the fluorescence intensity could decrease by $40-89 \%$ if the $\mathrm{pH}$ value of carbon dots solution changed from neutral to acidic or basic.

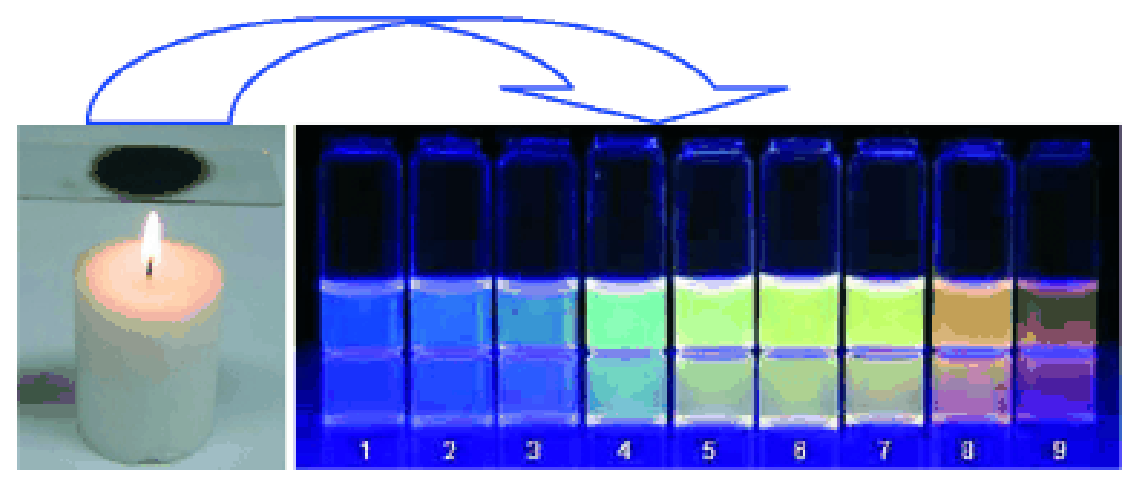

Figure 9. Water-soluble, multicolor fluorescent carbon nanoparticles are prepared by refluxing candle soot with nitric acid.

\subsubsection{Applications of CNPs}

On the basis of the unique fluorescent property, good aqueous solubility, low toxicity and photostability of CNPs, we will advance the discussion about the applications of CNPs in various fields, namely, bioimaging, nanoplatform therapy, heavy metal ions detection, fluorescent ink, explosive detection and light harvesting.

\subsubsection{Bio-imaging}

Fluorescent CNPs meet the prerequisites for bio-application, which typically requires materials with low toxicity, biocompatibility and photo-stability. Generally, the longer the emission wavelength of fluorescent carbon nanoparticles is, the better for bioimaging, in order to eliminate the effects of the autofluorescence in cells and tissue as well as the potential damage to the cells and tissue caused by short wavelength light.

Recently Cui et al. ${ }^{12}$ reported on the one-step synthesis of green fluorescent CNPs, which could be applied for the bio-imaging of live human HeLa cancer cells without 
extra surface modification. The excitation wavelength-dependent properties of the carbon dots were revealed by the fluorescence emission spectra, and the emission peaks located from 500-580 nm with the variation of excitation wavelength. The cytotoxicity of the CNPs was analyzed by treating the HeLa cells with different dose of as-synthesized CNPs ranging from $0-2 \mathrm{mg} / \mathrm{mL}$. Cell viability was not obviously decreased with the increase of the CNPs concentrations. 98\% cells were viable after 24 hours' incubation even though they were treated with high concentration of CNPs (2 $\mathrm{mg} / \mathrm{mL}$ ). After direct treatment of HeLa cells with the as-prepared CNPs, HeLa cells showed strong green fluorescence emission under blue light excitation (Figure 10), indicating that CNPs are an excellent fluorescent material for cell imaging.

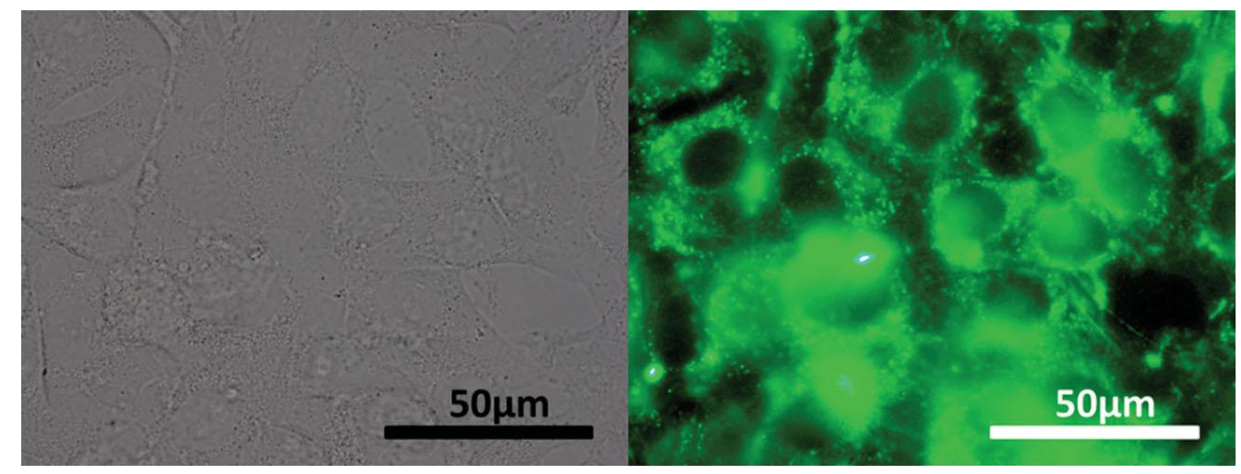

Figure 10. Bright field and green fluorescence microphotograph of HeLa cells treated with assynthesized CNPs.

Many other research groups have also been exploiting the applications of CNPs in bio-imaging. For instance, Gong et al. ${ }^{21}$ reported a facile and green synthetic route, which utilized pumpkin as the source under low-temperature $\left(90^{\circ} \mathrm{C}\right)$ hydrothermal treatment in the presence of $\mathrm{H}_{3} \mathrm{PO}_{4}$, to prepare phosphorous and nitrogen co-doped CNPs with the yellow fluorescence emission (Figure 11). The as-synthesized CNPs were demonstrated with the capabilities for cell imaging and real-time tracking of the $\mathrm{pH}$ variation in cells. Recently Shi et al. ${ }^{3}$ also prepared CNPs with dual-emission using 
naked oats through pyrolysis and microwave-assisted treatment. The biocompatible CNPs had the potential to be applied in bio-imaging of living cells. Jiang et al. ${ }^{22}$ used hydrothermal method to produce a yellow fluorescent CNPs from 1,2,4-triaminobenzen. The as-prepared CNPs not only were applied in cell imaging, but also displayed bifunctional sensing property, which can be used for monitoring $\mathrm{Ag}^{+}$in aqueous solution and Cys in human plasma sensing.
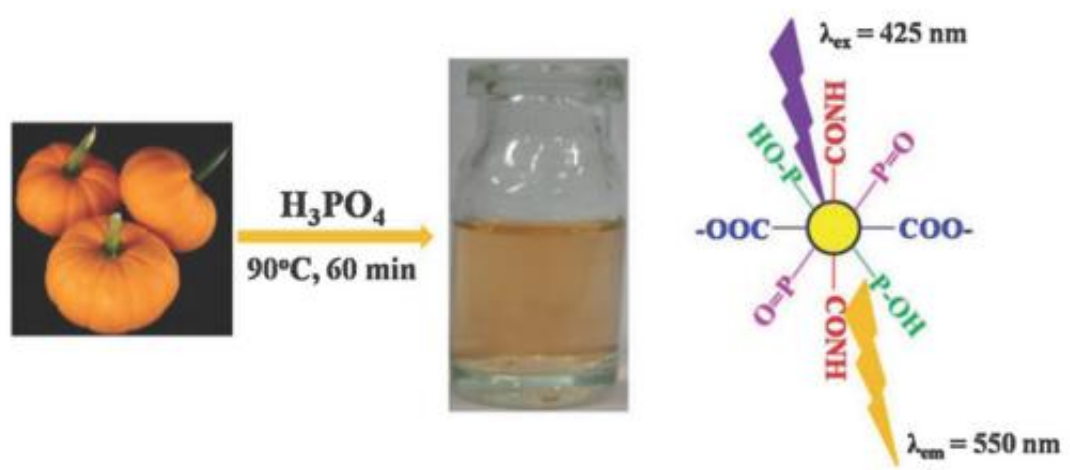

Figure 11. Scheme of CNPs synthetic route and the proposed structure of as-synthesized CNPs derived from pumpkin.

\subsubsection{Biomolecular sensing}

Biologically meaningful molecules, such as proteins, phytic acid or other small molecules in cells, play important roles in the physiological process. The abnormal level of these molecules in cells could lead to diseases. Consequently, there is an urgent need to develop selective and sensitive probes for monitoring concentration variation of these molecules.

Mercapto biomolecules including cysteine (Cys), homocysteine (Hcy), and glutathione (GSH) were regarded as important indexes for human health due to their important roles in cellular antioxidant defense systems and maintaining biological redox homeostasis. Recently a CNPs based ratiometric fluorescence probe for mercapto 
biomolecules sensing was developed by Lan's group. ${ }^{23}$ The CNPs with $30 \%$ quantum yield were prepared by employing melamine and trisodium citrate as precursors through a microwave-assisted hydrothermal method. The sensing probe for biothiols was designed by first adding $2 \mathrm{mM} \mathrm{Hg}^{2+}$ into CNPs solution in an appropriate ratio, and thus the blue fluorescence of CNPs was fully quenched by the addition of mercury solution due to electrostatic interaction between the surface functional groups (ammonia, hydroxyl group and carboxylic group) on CNPs and $\mathrm{Hg}^{2+}$ (Figure 12). The aforementioned non-fluorescent $\mathrm{CNPs} / \mathrm{Hg}^{2+}$ complex system could be applied for sensing biothiols via the tunable fluorescence of CNPs. The quenched fluorescence by $\mathrm{Hg}^{2+}$ could be rapidly recovered by addition of the mercapto biomolecules in 10 seconds because the strong reaction between $\mathrm{Hg}^{2+}$ and thiol group, thus resulting in the fluorescence "turn-on" of CNPs. The biocompatibility of CNPs/ $/ \mathrm{Hg}^{2+}$ complex was also investigated by treating the A549 cells with different concentration of CNPs/ $/ \mathrm{Hg}^{2+}$ complex prepared using CNPs in the range of $0-500 \mu \mathrm{g} / \mathrm{mL}$ of C-dos and $10 \mu \mathrm{M} \mathrm{Hg}^{2+}$. The viability of the A549 cells does not show an obvious decrease in comparison to that of the control group (without addition of CNPs/ $/ \mathrm{Hg}^{2+}$ ), indicating that the low toxicity of designed CNPs/ $\mathrm{Hg}^{2+}$ complex and its high potential for biothiols sensing in vivo. When living A549 cells were incubated with CNPs/ $/ \mathrm{Hg}^{2+}$, no fluorescence signal was observed. By further adding Cys into the culture media, the Cys was uptaken by cells and interacted with $\mathrm{Hg}^{2+}$, thus turning on blue fluorescence of CNPs. The limit of detection (LOD) of the fluorescence "turn-on" prober for biothiols is $15 \mathrm{nM}$. The probe also shows high selectivity against other common metal ions and amino acids without thiol group, 
indicating that the as-prepared CNPs offers an excellent sensing platform for sensitive and selective detection of metapto thiols.

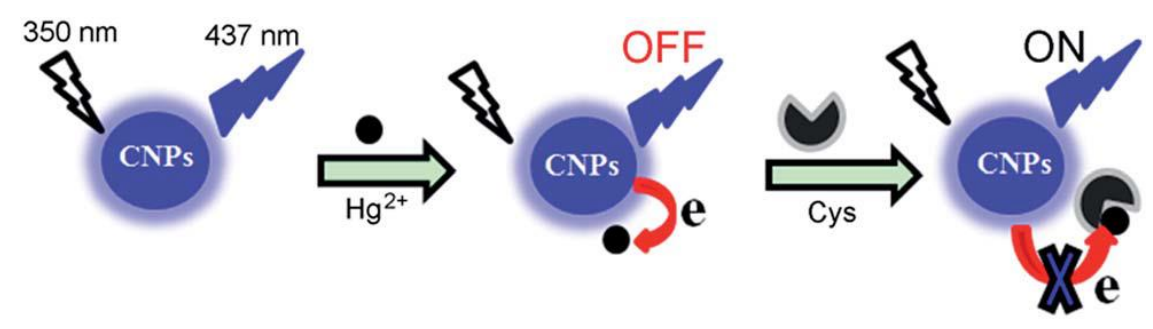

Figure 12. Schematic diagram showing the fabrication process of the CNPs/ $/ \mathrm{Hg}^{2+}$ sensor. The fluorescence of the sensor can be turned on by addition of Cys.

Several other fluorescence "turn off" or "turn-on" systems for biologically meaningful molecules based on the tunable PL property of CNPs have also been reported in recent years. Gao et al. ${ }^{4}$ reported a C-dot based fluorescent probe for phytic acid sensing. In this study, the CNPs were prepared using citric acid and lysine as carbon source and surface passivation reagent, respectively, under hydrothermal treatment in autoclave. The obtained CNPs emitting bright blue fluorescence was fully quenched by ferric ions to form the $\mathrm{CNPs} / \mathrm{Fe}^{3+}$ complex, which could serve as the sensing probe. The fluorescence of the biocompatible and low toxic $\mathrm{CNPs} / \mathrm{Fe}^{3+}$ complex would be recovered in the presence of phytic acid, which can be correlated to the concentration of phytic acid (Figure 13). The CNPs/ $/ e^{3+}$ based probe could detect phytic acid as low as $0.36 \mu \mathrm{M}$ with high sensitivity and selectivity. Recently Wang et al. ${ }^{24}$ investigated a class of N,S- co-doped fluorescent CNPs, which employed ammonium persulfate, glucose, and ethylenediamine as precursors and prepared under hydrothermal conditions. The obtained CNPs were able to detect the methotrexate in human serum with the detection limit of $0.33 \mathrm{nM}$ based on fluorescence quenching property. All these sensing application opens a new research direction for CNPs. 


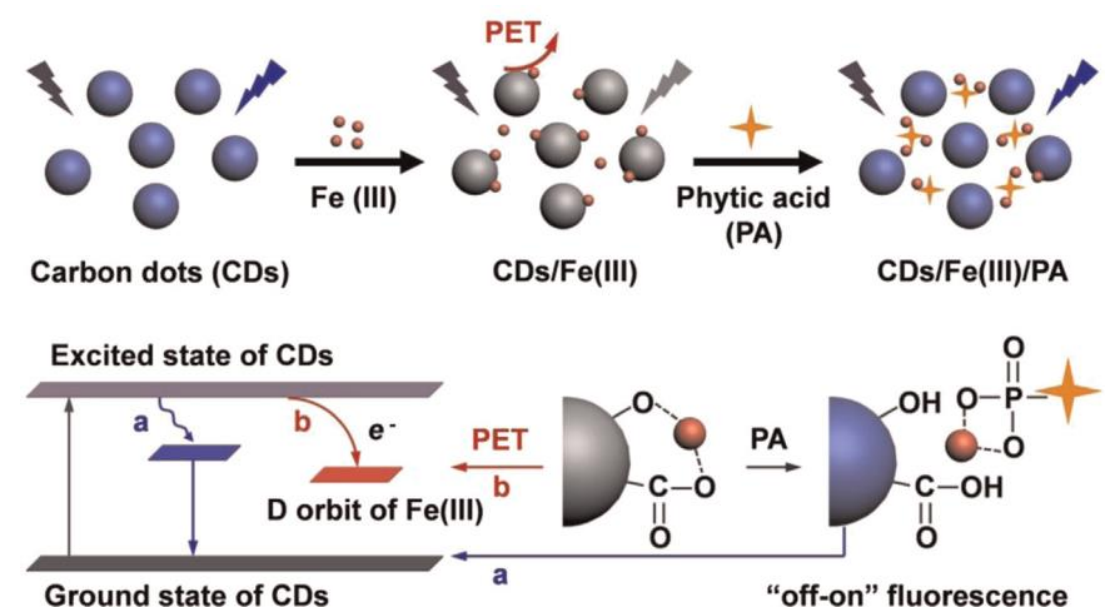

Figure 13. Schematic illustration of fluorescence response of CNPs in the presence of Fe (III) ions and Fe (III)/PA. Mode a: photo-induced fluorescence emission yielding bright blue luminescence. Mode b: photo-induced electron transfer (PET) in which an electron in the excited state enters the unfilled $\mathrm{d}$ orbit of $\mathrm{Fe}$, leading to fluorescence quenching of the CNPS.

\subsubsection{Phototherapy}

Phototherapy has received specific attention in recent years, due to its minimal invasiveness and the realization of localized treatment. Generally, there are two categories of phototherapy for cancers treatment, namely photodynamic therapy (PDT) and photothermal therapy (PTT). In PDT treatment, photosensitizers (PS) are used as PDT agents to react with the oxygen in tissue under laser irradiation, thus generating reactive oxygen species (ROS) to kill cancer cells, while in PTT treatment, PS are excited by infrared wavelength light and release vibration energy. Consequently, the heat produced by the excited PS could kill the target cells.

Ge et al. ${ }^{6}$ reported a novel fluorescent CNPs based nanomedicine, which combined the therapy function of PDT and PTT for cancer treatment. The precursors of CNPs were synthesized from the thiophene benzoic acid through a series of reactions, forming polythiophene phenylpropionic acid. The obtained dark red polythiophene phenylpropionic acid polymers were then dissolved in alkaline solution to produce CNPs 
through hydrothermal method. The as-synthesized CNPs emitted bright red fluorescence, with the emission wavelengths ranging from 640 to $680 \mathrm{~nm}$. The cytotoxicity of the CNPs and its application in PDT/PTT were analyzed in vitro by incubating B16-F0 skin cancer cells with as-synthesized CNPs followed by laser irradiation under different powder densities (Figure 14). There was no cell death observed in the presence of $200 \mu \mathrm{g} / \mathrm{mL}$ of CNPs or in the absence of CNPs but under laser irradiation ( 0.1 or $2 \mathrm{~W} \mathrm{~cm}^{-2}$ ), indicating the low toxicity of the as-prepared CNPs and the negligible killing effect of laser-produced heat on the viability of cancer cells. However, in the presence of CNPs and under irradiation of $635 \mathrm{~nm}$ laser with low power density $\left(0.1 \mathrm{~W} \mathrm{~cm}^{-2}\right)$, a portion of cells died due to the sole effect of PDT. More interestingly, in the presence of CNPs and under irradiation of $635 \mathrm{~nm}$ laser with high power density $\left(2 \mathrm{~W} \mathrm{~cm}^{-2}\right)$, all cancer cells were killed, accompanying the increase of temperature in the incubated cells solution up to $50^{\circ} \mathrm{C}$ within 5 mins. This result strongly supports the killing effect to cancer cells can be ascribed to the synergistic effects of PDT and PTT when higher power density laser was used. The in vivo therapy efficacy of CNPs was further investigated using HeLa-bearing nude tumor mice model. After the injection of $100 \mu \mathrm{L}$ of $2 \mathrm{mg} / \mathrm{mL}$ CNPs dissolved in PBS, the cancer cells were partially destroyed and slightly delayed increase of the tumor size was observed under lower power laser irradiation $\left(0.1 \mathrm{~W} \mathrm{~cm}^{-2}\right)$, which was consistent with in vitro result. This result also indicated that PDT therapy itself is insufficient to kill cancer cells. Alternatively, after localized treatment using higher power laser irradiation $\left(2 \mathrm{~W} \mathrm{~cm}^{-2}\right)$, all cancer cells were damaged. The therapy efficacy of PDT was evaluated to be $27 \%$ using the ROS $\left({ }^{1} \mathrm{O}_{2}\right)$ QY analysis, and the PTT conversion efficiency could reach 
$36.2 \%$. Further histological analysis of the treated mice shows that there was no

damage observed in main organs. All these features suggest that the as-synthesized

CNPs hold great potential in cancer phototherapy.
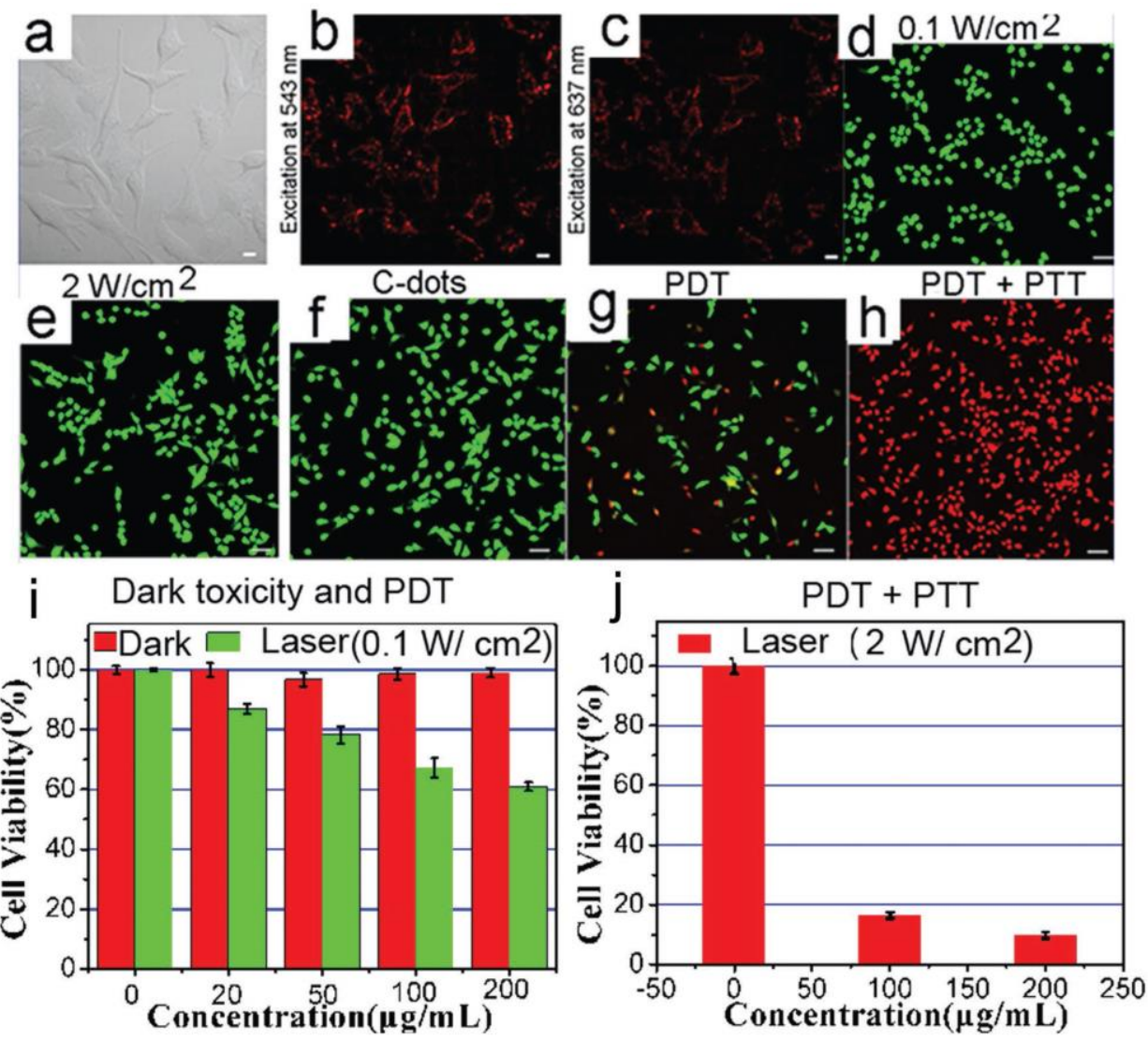

Figure 14. In vitro imaging and PDT/PTT. Confocal microscopy images of B16-F0 skin cancer cells incubated with CNPs (20 $\left.\mathrm{g} \mathrm{mLL}^{-1}\right)$ for 4 hat bright field a), Ex $=543 \mathrm{~nm} \mathrm{~b}$ ), and Ex $=637 \mathrm{~nm} \mathrm{c}$ ). Scale bar $=10 \mu \mathrm{m}$. Fluorescence images of calcein AM/PI-stained B16-F0 skin cancer cells incubated with various media: d) laser only $\left(0.1 \mathrm{~W} \mathrm{~cm}^{-2}\right)$, e) laser only $\left(2 \mathrm{~W} \mathrm{~cm}^{-2}\right)$, f) CNPs only $\left.\left(200 \mu \mathrm{g} \mathrm{mL}^{-1}\right), \mathrm{g}\right)$ $200 \mu \mathrm{g} \mathrm{mL} \mathrm{L}^{-1}$ of CNPs + laser (635 nm, $0.1 \mathrm{~W} \mathrm{~cm}^{-2}$, PDT), and h) $200 \mu \mathrm{g} \mathrm{m}^{-1}$ of CNPs + laser (635 nm, 2 $\left.\mathrm{W} \mathrm{cm}{ }^{-2}, \mathrm{PDT} / \mathrm{PTT}\right)$. Scale bar $=100 \mu \mathrm{m}$. Relative viability of B16-F0 skin cancer cells incubated with various concentrations of CNPs under dark or irradiation by $635 \mathrm{~nm}$ laser at power densities of i) $0.1 \mathrm{~W}$ $\mathrm{cm}^{-2}$ (PDT) and j) $2 \mathrm{~W} \mathrm{~cm}^{-2}$ (PDT/PTT) for $10 \mathrm{~min}$.

\subsubsection{Heavy metal ions detection}

Tiny amount of the metal ions change in human boy will affect physiological

activities. In addition, some heavy metal ions are highly toxic to cells and tissues.

Therefore, in recent years, researchers have engineering CNPs through surface 
functionalization during synthesis and then developed fluorescent CNPs based probes for selective and sensitive metal ions detection. Surface functionalization of the CNPs has been realized during the CNPs synthesis through different doping strategies (e.g., nitrogen, sulfide or phosphor doping). The electron-donating elements not only enhance the PL of CNPs, but also endow them the capability for metal ions detection. In addition, those fluorescent CNPs could possess good selectivity and selectivity for metal ions due to abundant carboxyl groups, hydroxyl groups and nitrogen groups on the surface of CNPs. The fluorescence of the CNPs can be quenched through the electron transfer process between the functionalized CNPs and the electron-deficient heavy metals ions (e.g. $\mathrm{Hg}^{2+}, \mathrm{Fe}^{3+}$, and $\mathrm{Cu}^{2+}$ ) even at very low concnetration. ${ }^{25-28}$

Gong et al. ${ }^{21}$ reports the nitrogen-doped CNPs for ferric ions detection. Chitosan, acetic acid and 1, 2-ethylenediamine (EDA) were utilized as carbon and nitrogen sources under pyrolysis in the domestic microwave oven. The dark brown solution of the CNPs emitted strong blue fluorescence under UV-light and the maximum PL intensity of emission peak was at around $440 \mathrm{~nm}$. The sensitivity and selectivity of CNPs for $\mathrm{Fe}^{3+}$ were demonstrated by titrating the as-prepared CNPs aqueous solution $(0.10 \mathrm{mg} / \mathrm{mL})$ with different $\mathrm{Fe}^{3+}$ concentration and other common metal ions at $\left.10 \mathrm{mM}\right)$, respectively. The PL intensity of the CNPs was not obvious changed except for slightly decreased by $\mathrm{Fe}^{2+}, \mathrm{Cu}^{2+}, \mathrm{Pb}^{2+}, \mathrm{Hg}^{2+}, \mathrm{Ag}^{2+}, \mathrm{Cd}^{2+}$ and $\mathrm{Cr}^{3+}$ ions, compared with that was fully quenched by $\mathrm{Fe}^{3+}$ (Figure 15). The detection limit for $\mathrm{Fe}^{3+}$ ion could reach as low as $10 \mathrm{ppb}$. The biocompatibility of the as-synthesized CNPs was investigated through standard MTT assay, and the CNPs also showed good performance for $\mathrm{Fe}^{3+}$ sensing in cells. 
(A)

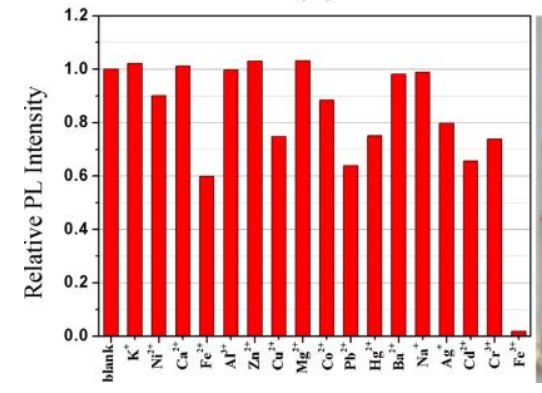

(B)

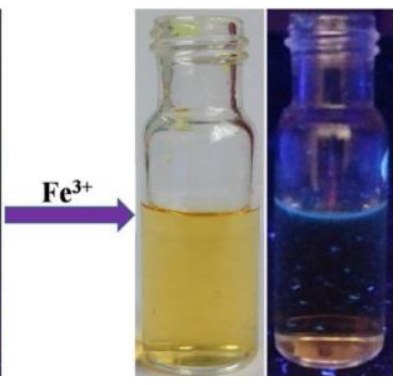

Figure 15. (A) The relative change in PL intensity of $N-C D s(0.10 \mathrm{mg} / \mathrm{mL})$ after reacting with different metal ions $(10 \mathrm{mM})$. (B) Photographic images of $\mathrm{N}-\mathrm{CDs}(0.10 \mathrm{mg} / \mathrm{mL})$ before and after adding $\mathrm{Fe}^{3+}(280 \mathrm{ppm})$ under daylight (left panel) and UV irradiation (right panel).

Recently Wen et al. ${ }^{29}$ also prepared CNPs using cotton through pyrolysis and microwave methods. The as-prepared blue fluorescent CNPs showed high selectivity and sensitivity toward $\mathrm{Fe}^{3+}$ detection with a limit of detection as low as $2.7 \times 10^{-8} \mathrm{M}$. Other CNPs based probes for $\mathrm{Fe}^{3+}$ sensing have also been reported in literatures. For instance, Jiang et al. ${ }^{7}$ reported $\mathrm{N}$-doped CNPs that was produced from citric acid and isoleucine using hydrothermal method. The as-synthesized CNPs showed high selectivity and sensitivity for $\mathrm{Fe}^{3+}$ compared with other competitive metal ions. Beyond $\mathrm{Fe}^{3+}$ detection, it is also important to detect $\mathrm{Hg}^{2+}$ ions due to its high toxicity and frequent occurrence in water system. Li et al. ${ }^{30}$ demonstrate that their synthesized CNPs possessed high selectivity against other interference ions and the limit of detection for $\mathrm{Hg}^{2+}$ was as low as $10 \mathrm{nM}$. Lan et al. ${ }^{31}$ also reports the CNPs based ratiometric fluorescent sensor for $\mathrm{Hg}^{2+}$ detection in buffer solution and living cells. Additionally, Liu et al. ${ }^{32}$ reported a green route to synthesize CNPs under hydrothermal treatment of grass. The obtained N-doped CNPs exhibited high sensitivity for $\mathrm{Cu}^{2+}$ ions detection. Jiang et al. ${ }^{22}$ reported the yellow emissive CNPs synthesized from 1,2,4Triaminobenzene hydrothermally and its application in $\mathrm{Ag}^{+}$sensing. Furthermore, Qu et al. ${ }^{33}$ investigated the possibility to differentiate multiple metal ions using dual-emission 
CNPs and the results show that the prepared CNPs could successfully distinguish $\mathrm{Fe}^{3+}$, $\mathrm{Bi}^{3+}$ and $\mathrm{Al}^{3+}$ due to the fluorescence quenching in different wavelength regions.

\subsubsection{Fluorescent ink and information storage}

Fluorescent CNPs used as fluorescent ink was rarely reported in previous literatures because of the limitations such as low quantum yield of fluorescent CNPs and selfquenching of high dose of CNPs via aggregation formation.

Recently Qu's group ${ }^{15}$ reported dual fluorescence emission of CNPs, which can be used as fluorescence ink. The fluorescent CNPs were synthesized using citric acid and urea as precursors through one-step microwave-assisted method. The obtained brown fluorescent CNPs solution showed excitation-wavelength-dependent PL property. The emission peaks located from 420 to $570 \mathrm{~nm}$, which are responsible to blue, green and red emission colors. In addition, when the CNPs aqueous solution coated on the commercially available lens paper, the excitation-wavelength-dependent PL property could also be maintained, and the maximum fluorescence intensity of the CNPs coated on the paper was enhanced by $40 \%$, indicating that the as-synthesized CNPs can be used as fluorescence ink. As shown in Figure 16, characters "395" were written on the lens paper with CNPs solution and then closed the "circles "of 395 with commercial green fluorescent ink to result in characters "888". Under blue UV-light irradiation, the green fluorescent number "888" appeared on the paper. Under green UV-light, the green fluorescent ink was invisible and only "395" written with CNPs solution was observed with red fluorescence. Based on the dual-fluorescence-emission property, the integrated system combining CNPs and commercial green fluorescent ink may provide a new route in counterfeiting and information encryption. 
a)

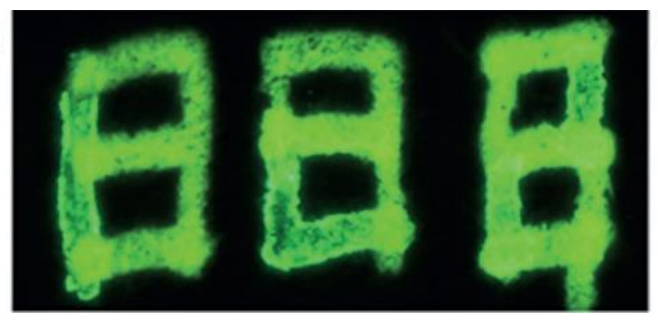

b)

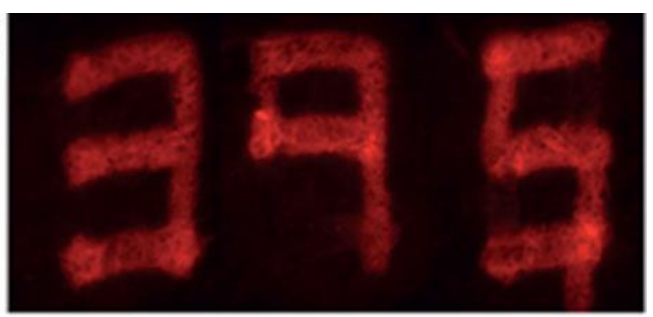

Figure 16. Fluorescent characters on commercially available lens paper under different excited UV-light. a) Characters 888 written by commercial green fluorescent ink and as-synthesized CNPs under blue UV-light; b) just characters 395 labeled with CNPs solution showed red fluorescence under green UV-light.

The fluorescent CNPs with high quantum yield were also utilized as fluorescent ink for pattering and painting purpose. Wen et al. ${ }^{29}$ reported the fluorescent CNPs derived from cotton for multicolor pattering. The obtained CNPs aqueous solution was drawn on commercially available papers without UV fluorescence background. The CNPs labeled area on papers showed blue and green fluorescent patterns under 365 and $470 \mathrm{~nm}$ excitation, respectively. In addition, the bright blue and green patterns still could be observed after several months, demonstrating the excellent photostability of CNPs. Wang et al..$^{34}$ developed a simple and large-scale preparation route of CNPs. The excitation-wavelength-dependent PL behavior enabled the as-synthesized CNPs to be used as printing inks. The as-prepared CNPs aqueous sample was filled in a commercial inkjet printer and the printed words and images showed green and red fluorescence under 455 and $523 \mathrm{~nm}$ excitation, respectively, as shown in Figure 17. Its application as fluorescent ink was also demonstrated using other substrates such as silk fibers and silk fabric. 

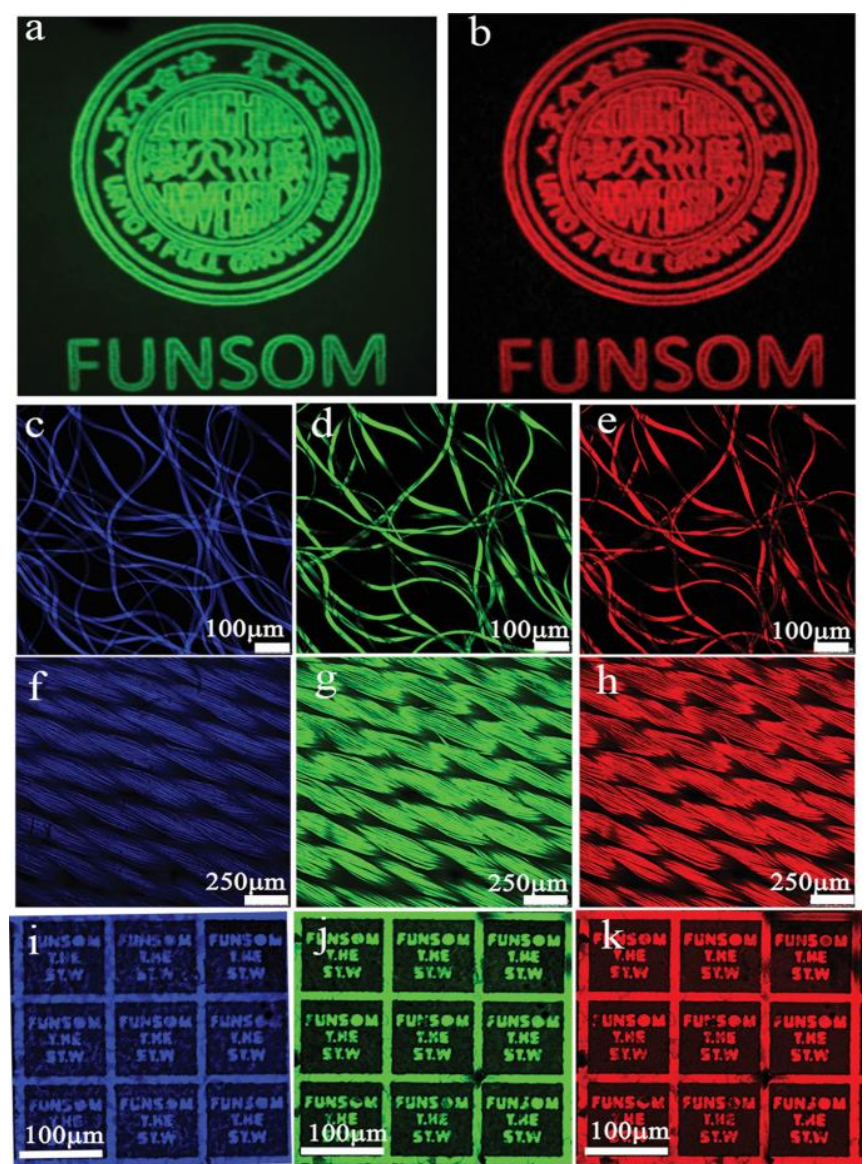

Figure 17. $(a, b)$ Fluorescent images on commercially purchased paper stained with the C-dot ink captured by the CRI Maestro in vivo imaging system with excitation at $455 \mathrm{~nm}$, emission at 500-600 nm, and exposure time $30 \mathrm{~ms}$ (a) and excitation at $523 \mathrm{~nm}$, emission at 560-650 nm, and exposure time 75 ms (b). (c-e) Fluorescence images of the C-dot-stained silk fibers: (c) $\lambda$ excitation $=405 \mathrm{~nm}$; emission band pass: $420-480 \mathrm{~nm}$; (d) $\lambda$ excitation $=488 \mathrm{~nm}$; emission band pass: $500-580 \mathrm{~nm}$; (e) $\lambda$ excitation $=$ $543 \mathrm{~nm}$; emission band pass: 580-660 nm. (f-h) Fluorescence images of the CNPs-coated silk fabric: (f) $\lambda$ excitation $=405 \mathrm{~nm}$; emission band pass: $420-480 \mathrm{~nm}$; $(\mathrm{g}) \lambda$ excitation $=488 \mathrm{~nm}$; emission band pass: $500-580 \mathrm{~nm}$; (h) $\lambda$ excitation $=543 \mathrm{~nm}$; emission band pass: $580-660 \mathrm{~nm}$. (i-k) C-dot ink is drop-cast on a hydrophilic photolithography pattern: (i) $\lambda$ excitation $=405 \mathrm{~nm}$; emission band pass: $420-480 \mathrm{~nm}$; (j) $\lambda$ excitation $=488 \mathrm{~nm}$; emission band pass: $500-580 \mathrm{~nm}$; $(\mathrm{k}) \lambda$ excitation $=543 \mathrm{~nm}$; emission band pass: 580-660 $\mathrm{nm}$. All the pictures are imaged with a laser-scanning confocal microscope (LSCM).

\subsubsection{Explosive detection}

Instant, sensitive and selective detection of explosives has gained increasing attention in recent years because of the implications in homeland security, global demining and environmental safety. Although trinitrotoluene (TNT) is the most widely used military explosives, picric acid (PA, also known as 2,4,6-trinitrophenol) is regarded as an even more violent explosive than the well-known TNT. Therefore, PA has been 
widely used in the preparation of lethal weapons, manufacture of rocket fuels, dyes, fireworks and matches. In addition, the wide use of PA could contaminate our environment and thus PA has been recognized as an environmental pollutant. Consequently, it is highly demanded to develop novel sensory material for highly sensitive and selective picric acid detection. Among all sensing technologies, fluorescence-based explosive detection possesses the advantages of simple operation, cost effectiveness, quick response and high sensitivity.

Recently our group developed an ultrafast synthetic route to produce fluorescent CNPs by employing ammonium citrate dibasic (ACD) as single precursor which was further applied for picric acid (PA) sensing. ${ }^{16}$ The obtain CNPs solution showed excitation-wavelength-independent behavior and its blue fluorescence emission could be sensitively and selectively quenched by picric acid explosive. Upon addition of different concentrations of PA solutions in the range of $0-100 \mu \mathrm{M}$ (Figure 18), the fluorescent intensity of the CNPs solution was gradually quenched, while other nitroexplosives (TNT, DNT, 2-NT, DNB and NM) affected the fluorescence intensity insignificantly, indicating good selectivity in PA detection. The sensing mechanism was systematically investigated and the synergistic effect of its low molecular orbitals, the presence of fluorescence resonance energy transfer as well as acid-base interactions between picric acid and fluorescent CNPs was believed to contribute to the excellent sensing performance to picric acid. This study demonstrated a simple way to prepare highly fluorescent CNPs and also broadened the application of CNPs. 

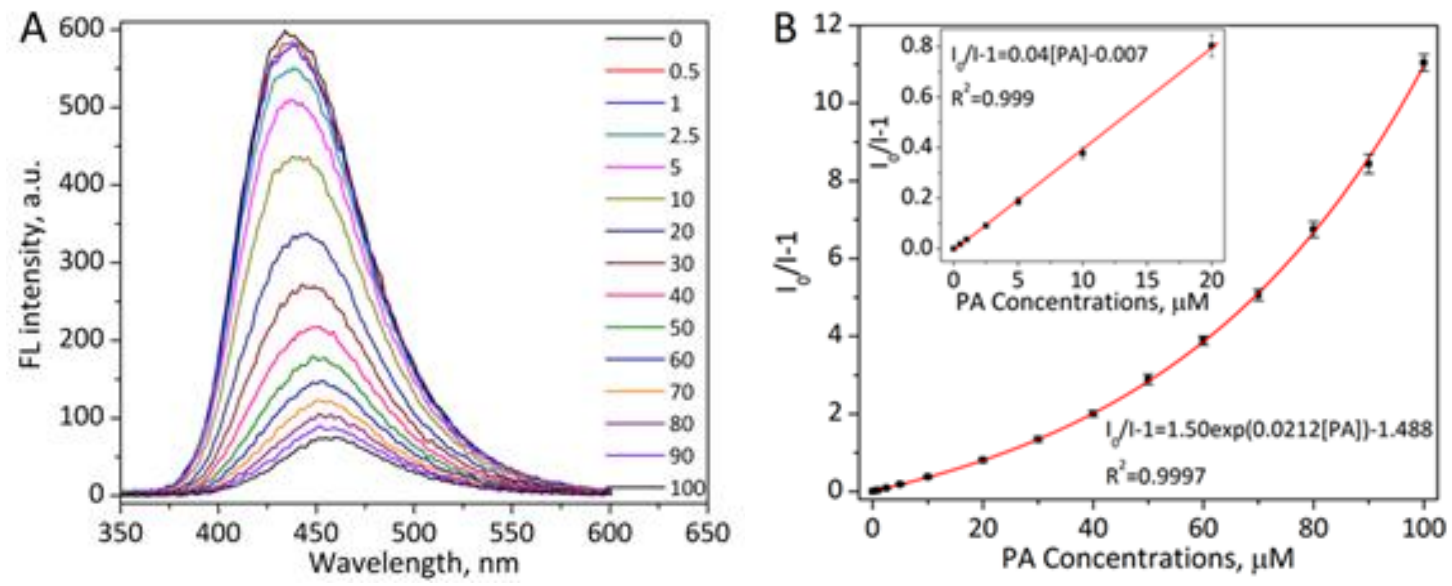

Figure 18. (A) Fluorescence spectra of CNPs upon addition of various PA concentrations $(0-100 \mu \mathrm{M})$ in water. (B) Fitting curve of the nonlinear Stern-Volmer plot for PA by exponential quenching equation. Inset shows the fitting curve of the linearity of Stern-Volmer plot for PA of 0 $20 \mu \mathrm{M}$.

Obtaining fluorescence emission with different colors has been considered as a key requirement for successfully utilizing carbon dots in various applications. So far most of carbon dots emit blue color under ultraviolet excitation. Although the excitationdependent fluorescence emission has been reported, a significant decrease in fluorescent intensity was also observed with changing of excitation wavelength. Researchers are trying to develop synthetic methods to shift the maximum emission light from blue, to green, yellow, and red in order to broaden the applications of CNPs. However, most of current synthesis endured issues such as complex procedures, toxic components or low quantum yield. It is still difficult to synthesize CNPs through a facile strategy with different fluorescence emission and high quantum yield in one single material. The emission of CNPs could be tuned by controlling the dehydration reaction, chemical manipulation, doping of other elements. Nitrogen is by far the most prominent doping candidate into carbon to obtain fluorescent CNPs. Doping the carbon materials with other heteroatoms such as boron (B), and sulfur (S) has also been reported. In 
another work in our group, we report a novel carbon dots with high quantum yield and dual fluorescence emission ${ }^{9}$. The CNPs were synthesized using nitrogen and phosphorous co-doped carbon sources through one simple and fast microwaveassisted method. The as-synthesized nitrogen and phosphorus co-doped CNPs (N, PCNPs) were thoroughly characterized using various characterization techniques such as TEM, XRD, FTIR, XPS, etc. The optical results suggest that more than one type of excitation energy trapped on the surface of CNPs and the CNPs demonstrated excitation-dependent and $\mathrm{pH}$-independent emission properties. Especially, under different excitation wavelengths, the CNPs showed blue and green emission, the quantum yield of which are 0.51 and 0.38 , respectively. The nitro-explosives detection through the quenching of different emission were investigated, in which picric acid quenched both blue and green emission, while TNT only quenched green emission to some extent (Figure 19). We expect that the as-prepared CNPs provide new insights into the development of a ratiometric sensor to distinguish different explosives because of their dual fluorescence emission.
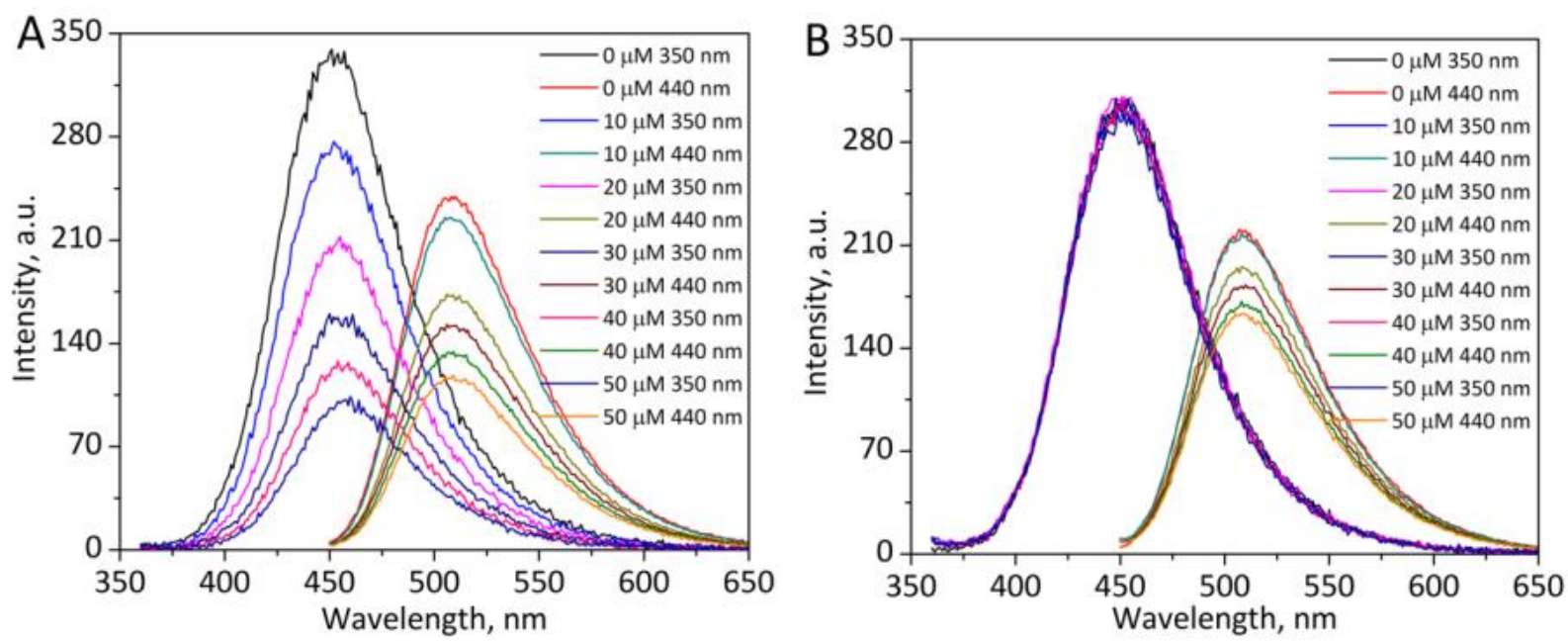
Figure 19 Explosives detection using diluted CNPs solutions with incremental addition (0 $50 \mu \mathrm{M})$ of (A) picric acid and (B) TNT, respectively.

Additionally, Krishna et al. ${ }^{35}$ introduced a boron and nitrogen co-doped CNPs (BN-CNPs) for selective and sensitive detection of picric acid (Figure 20). The BN-CNPs with strong photoluminescence and a quantum yield of $\sim 14.2 \%$ was produced by a hydrothermal method using a mixture of sucrose, boric acid, and urea as the precursor. Its application for selective and sensitive detection of picric acid (PA) was realized through remarkable photoluminescence signal quenching upon the interaction between PA and CNPs. The good selectivity was also demonstrated with insignificant interference from other common explosives. PA as low as $10 \mathrm{nM}$ can be detected at room temperature and $\mathrm{pH} 7$.

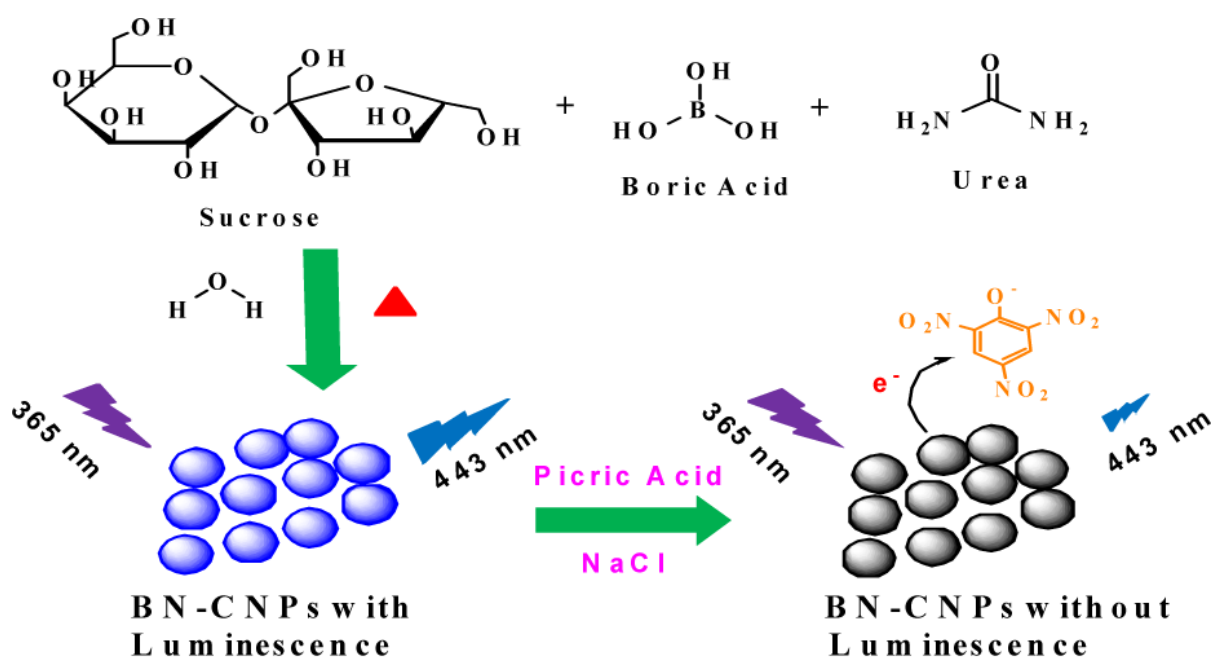

Figure 20. The synthesis of BN-doped CNPs and its application for picric acid detection.

\subsubsection{Light harvesting}

As a new application, CNPs were also employed in light harvesting ${ }^{11}$. Nitrogenenriched CNPs with diameter of 1 to $3 \mathrm{~nm}$ and fluorescent quantum yield of up to $22 \%$ were produced through a one-pot reaction between melamine and glycerol in an 
elevated temperature. The N-rich CNPs were then applied to sensitize $\mathrm{TiO}_{2}$ based photoanode to study the conversion of near IR irradiation to photocurrent (Figure 21). The results indicate that the as-prepared N-doped CNPs display comparable performance as other conventional sensitizers such as CdSe and InAs quantum dots at their early development stage. As IR accounts for over $50 \%$ of solar energy, the developed CNPs hold great promise in light-harvesting application and it can be used as a complementary material to other photosensitizers, thus maximizing energy conversion efficiency.

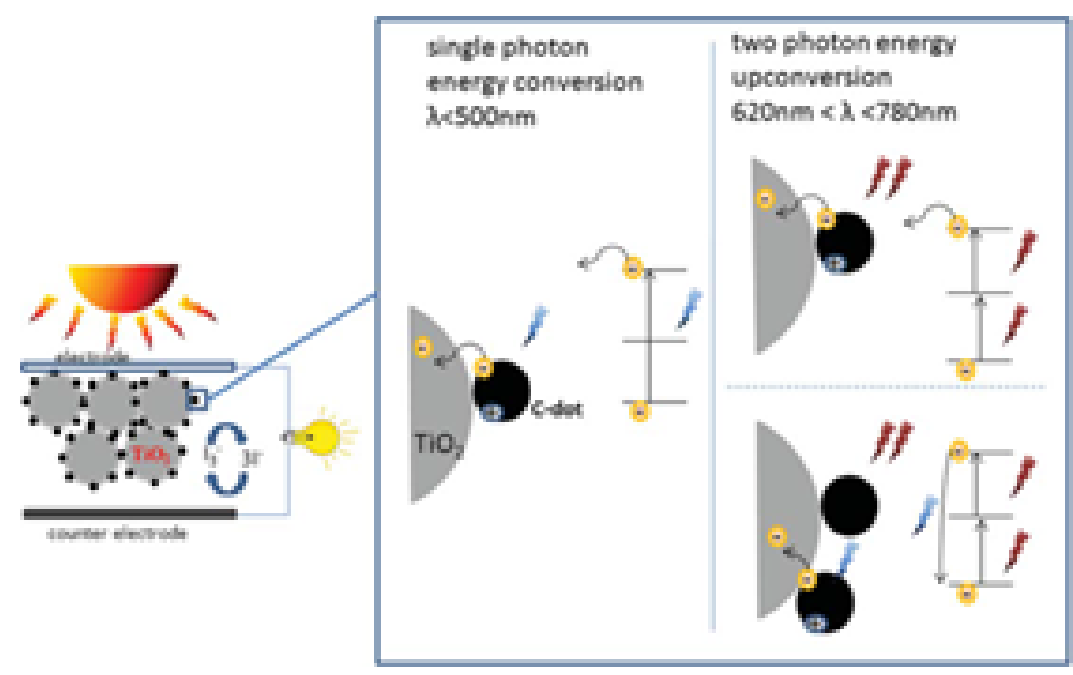

Figure 21. Working mechanisms of nitrogen-enriched CNPs as light harvesting agents for the $\mathrm{TiO}_{2}$ based photoanode.

\subsection{Palladium and its applications}

Palladium is an important group 10 element, which has been widely used in many fields including fuel cells, jewelry, medicine and automobile industry. ${ }^{36}$ Especially, Pd-catalyzed reactions exhibit high efficacy that are widely applied in medicine synthesis. Due to the extensive applications of palladium, it is highly possible for $\mathrm{Pd}^{2+}$ to migrate into the environment. The residual palladium can then be accumulated in food 
chain and bind with the biothiols, DNA, proteins and Vitamin B, which could impose significant risk on human health. ${ }^{37}$ For example, the U.S. federal agency restricts the threshold limit of final concentration of the palladium in drug chemicals in the range of 47.0 to $94.0 \mu \mathrm{M} .{ }^{38}$ Consequently, there exists an urgent need to develop a novel, sensitive and selective probe for palladium sensing. Although the typical analytical techniques, such as atomic absorption spectrometry, plasma emission spectroscopy, solid phase microextraction-high performance liquid chromatography, X-ray fluorescence, etc. meet the demand of quick analysis with high sensitivity, they are greatly limited by the high cost of instrumentation, poor portability and fieldibility, and requirement of highly trained individuals..$^{39}$

\subsection{Fluorescence-based palladium sensing}

Compared to afore-mentioned analytical methods, fluorescence-based detection possesses distinct benefits, including convenient operation, high sensitivity, costeffectiveness, excellent portability and rapid response. ${ }^{40}$ In general, there were two strategies employed for fluorescence-based palladium detection. One strategy for palladium sensing took the advantage of $\mathrm{Pd}$-catalyzed reactions, such as the $\mathrm{Pd}$ catalyzed cyclization of oxazole, pyrone, and pyrrole, the Pd-catalyzed depropargylation reaction and deallylation reaction, as well as the Pd-catalyzed Heck or the SuzukiMiyaura coupling reaction. For example, Wang et al. reported a sensitive chemosensor for $\mathrm{Pd}^{2+}$ detection using rhodamine $\mathrm{B}$ as precursor and the catalytic feature of $\mathrm{Pd}^{2+}$ in organic synthetic reactions. ${ }^{41}$ The fluorescence was "turned-off" due to the reaction of changing the conjugated structure to xanthene derivative. Upon addition of $\mathrm{Pd}^{2+}$, the fluorescence of the conjugated structure was recovered. In the other strategy, $\mathrm{Pd}^{2+}$ is a 
strong fluorescence quencher due to the fact that $\mathrm{Pd}^{2+}$ has a $\left(4 \mathrm{~d}^{8}\right)$ electronic configuration. Fluorescence-quenching based sensors for palladium detection are proposed through PET (photo-induced electron transfer) mechanism.

\subsection{Objective of thesis}

The objective of this study is to design a fluorescence-based chemosensor for $\mathrm{Pd}^{2+}$ sensing via a modified microwave-based synthetic route. The desirable probe displays excellent sensitivity and selectivity for $\mathrm{Pd}^{2+}$ detection. The simple synthetic route of the fluorescent CNPs and their excellent sensitivity and selectivity to $\mathrm{Pd}^{2+}$ detection provide a new route to the development of sensitive and selective fluorescent $\mathrm{Pd}^{2+}$ sensors. 


\section{DESIGN OF CNPS-BASED FLUORESCENCE PROBE FOR}

\section{PALLADIUM SENSING}

\subsection{Preparation methods of CNPs}

Carbon dots can be prepared by a number of methods but that all generally fall into two broad categories: bottom-up and top-down approaches. In bottom-up approaches, the CNPs are obtained from assembling molecular precursors under a range of different reaction conditions, including hydrothermal, microwave-assisted, ultrasonic, acid dehydration, and pyrolytic condition. CNPs could be prepared through "Top down" approaches from large cluster of carbon materials, such as carbon nanotubes, graphite, carbon soot and so on through arc-charge, laser ablation and chemical oxidation. Especially, the microwave-assisted method provides a low cost, rapid reaction rate and one-step synthetic route compared with other aforementioned methods. ${ }^{15}$

The surface functionalized strategy also have been investigated in the CNPs synthesis process through different doping strategies (e.g., nitrogen, sulfide or phosphor doping ${ }^{11}$. The doping heteroatoms not only enhance the PL property of CNPs as election-donating elements, but also act as ligands to interact with other components to detect target molecules sensitively and selectively.

2.2. The design of CNPs-based fluorescence chemosensor for $\mathrm{Pd}^{2+}$ sensing

a)

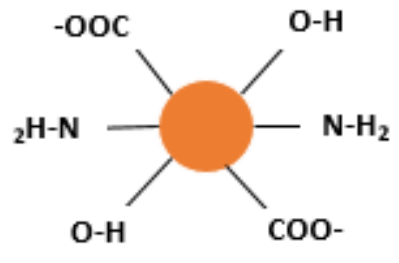

b) excitation

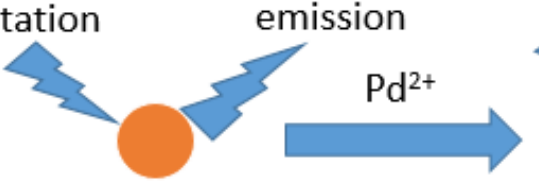

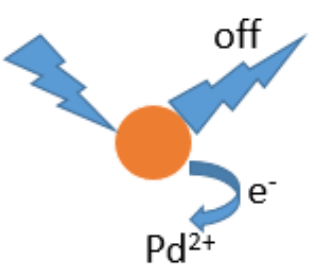


Scheme 1, Schematic diagram showing a) the proposed structure of the CNPs and b) the sensing mechanism of the CNPs-based probe for $\mathrm{Pd}^{2+}$ detection. The fluorescence of the sensor can be turned off in the presence of $\mathrm{Pd}^{2+}$.

The precursors are employed as carbon and nitrogen resources with hydrothermal treatment in the microwave oven. The proposed structure of the obtained CNPs is shown as scheme $1 \mathrm{a}$ ). The surface of the carbon core is functionalized with the hydrophilic chemical groups with the result of good water solubility and stability. In addition, CNPs also exhibits sensitivity for $\mathrm{Pd}^{2+}$ recognition due to the high affinity between the nitrogen and $\mathrm{Pd}^{2+}$.

The sensing mechanism of the CNPs-based probe for $\mathrm{Pd}^{2+}$ detection was illustrated in scheme1 b). The unique PL property of the CNPs that can be excited at a low wavelength and emit fluorescence in a longer wavelength. The fluorescence will be quenched in the presence of $\mathrm{Pd}^{2+}$ due to the photo-induced electronic transport process between the CNPs and $\mathrm{Pd}^{2+}$. 


\section{MATERIALS AND METHODS}

\subsection{Reagent and chemicals}

Citrate acid (anhydrous, 99.5\%), copper chloride (anhydrous, 99\%), magnesium chloride (hexahydrate, 99\%) and manganese nitrate (tetrahydrate, 99\%) were obtained from Acros Organics. Calcium chloride (dehydrate, 99\%), polyethyleneimine (50\% w/v in water, $M W=750,000$ ), iron chloride (hexahydrate, $97 \%$ ), nickel sulfate (hexahydrate, 299.99) and palladium chloride (anhydrous, 99\%) were bought from Sigma Aldrich. Cadmium nitrate (tetrahydrate, $\geq 99.0 \%$ ) was purchased from Fluka. Zinc chloride (anhydrous, 99.95\%) was obtained from Alfa Aesar. Cobalt chloride (hexahydrate, 98\%) was obtained from Fisher. All the other chemicals were of analytical grade. The glutinous polyethylenimine (50\% w/v in water) was diluted to $20 \% \mathrm{w} / \mathrm{v}$ of transparent solution for further use.

\subsection{Preparation of fluorescent carbon nanoparticles}

A modified microwave method ${ }^{42}$ was employed to synthesize fluorescent CNPs. Briefly, $1.0 \mathrm{~g}$ of Citric acid and $1 \mathrm{ml}$ of PEl $(20 \% \mathrm{w} / \mathrm{v})$ was dissolved in $2.3 \mathrm{ml}$ deionized water to form a homogeneous and transparent solution. The solution was then heated in the advanced microwave synthesizer (Discover SP, CEM) under $180^{\circ} \mathrm{C}$ and $300 \mathrm{~W}$ for 1h, during which the solution changed from colorless liquid to a dark brown aqueous solution. Upon cooling, an aqueous solution of the CNPs was purified in a centrifuge $(10,000 \mathrm{rmp}$ min-1, 30 mins) to remove any large or agglomerated particles and then dialyzed against water through the dialysis membrane $(0.5-1 \mathrm{KD})$ to remove the residues of small molecules. The final homogeneous solution was stable at room temperature for months. 


\subsection{Characterization}

A high resolution transmission electron microscope (HRTEM, JEOL 2010 TEM) at an operating voltage of $200 \mathrm{kV}$ was used to observe the morphology and microstructures of the CNPs. The X-ray photoelectron spectra (XPS) was obtained on a PHI model 590 spectrometer equipped with multiprobes ( $\Phi$ Physical Electronics Industries Inc.), using a monochromatic Al Ka X-ray source (hv = $1486.6 \mathrm{eV})$. The X-ray diffraction patterns were collected on a Rigaku Ultima IV instrument with $\mathrm{Cu} \mathrm{Ka}$ radiation operating at $40 \mathrm{KV}$ and $44 \mathrm{~mA}$. Fourier transformed infrared (FTIR) spectra were collected on a Nicolet 560 Fourier spectrometer with a Diamond ATR accessory. The absorption spectra were obtained using a Cary 50 UV-vis spectrophotometer (Agilent Technologies). Fluorescence excitation spectra and emission spectra were measured using a Varian Cary Eclipse fluorescence spectrometer (Agilent Technologies).

\subsection{Detection of $\mathrm{Pd}^{2+}$ in the aqueous phase}

The sensing experiments were carried out by monitoring the fluorescence quenching behavior of the fluorescence quenching behavior of the carbon nanoparticles solution upon the addition of target metal ions at room temperature through a fluorescence spectrometer, which excitation and emission slit widths of $5 \mathrm{~nm}$. Briefly, 3 $\mathrm{ml}$ diluted CNP solution ( $1 \mu \mathrm{L}$ of CNP solution diluted in $3 \mathrm{~mL}$ deionized water) was placed in a disposal cuvette and then gradually adding $\mathrm{Pd}^{2+}$ aqueous solution. Each titration was repeated at least three times and consistent results are reported. 
The fluorescence emission experiments were excited at $350 \mathrm{~nm}$, and the emission data were collected in the range of $361-600 \mathrm{~nm}$. The quenching efficiency (QE) was calculated as the following equation:

$$
\mathrm{QE}=1-/ / I_{0} \quad \text { Equation } 1
$$

Where $l_{0}$ is the initial fluorescence intensity of in the absence of the analyte and $I$ is the fluorescence intensity in the presence of the corresponding analyte. 


\section{RESULT AND DISCUSSIONS}

4.1. Morphology and characterizations of CNPs

4.1.1. High resolution of TEM

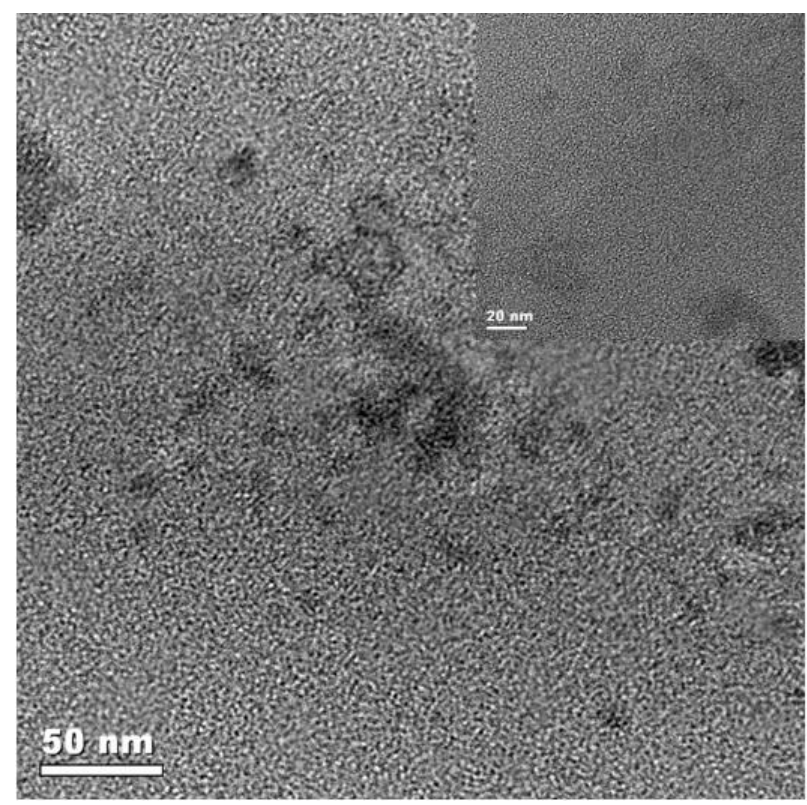

Figure 22. TEM graph of the CNPs and insert shows the TEM imaging of CNPs with $20 \mathrm{~nm}$ scale bar.

TEM imaging (Figure 22) reveals the amorphous structure of the as-synthesized CNPs which have an average diameter of 2-3 $\mathrm{nm}$ 


\subsubsection{X-ray diffraction pattern}

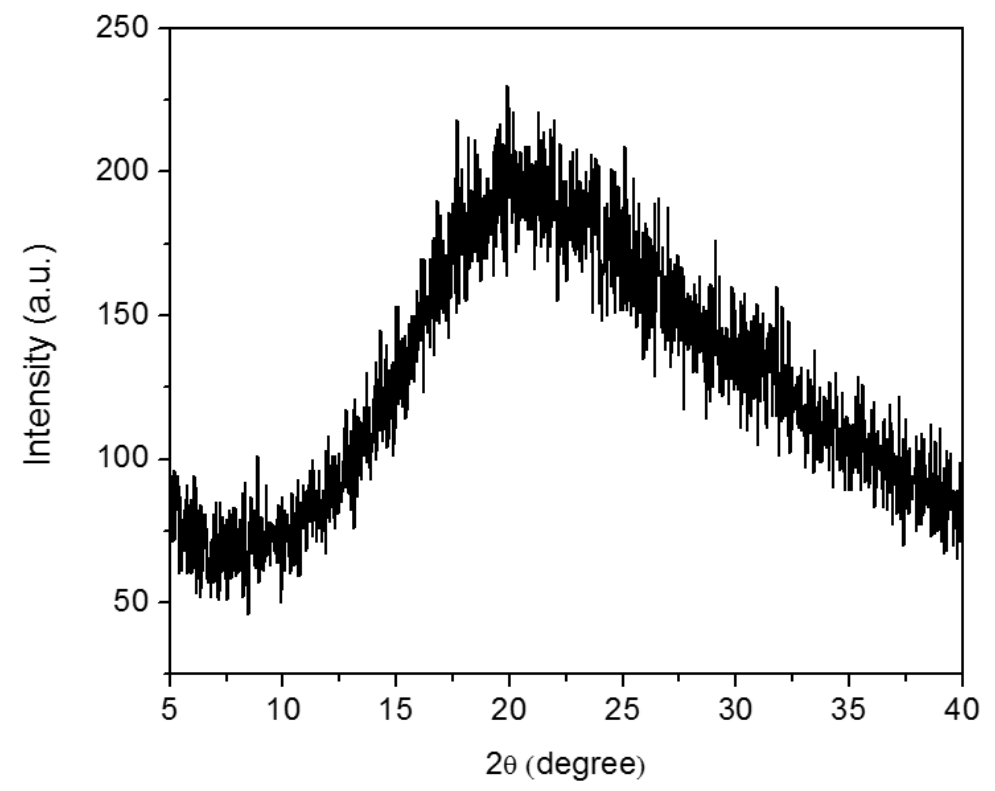

Figure 23. XRD pattern of the CNPs

As shown in Figure 23, the XRD pattern of the CNPs shows a broad peak centered at around $20^{\circ}$, which also corroborates the amorphous feature of the CNPs, in good agreement with the TEM results.

\subsubsection{Fourier transformed infrared spectrum}

c)

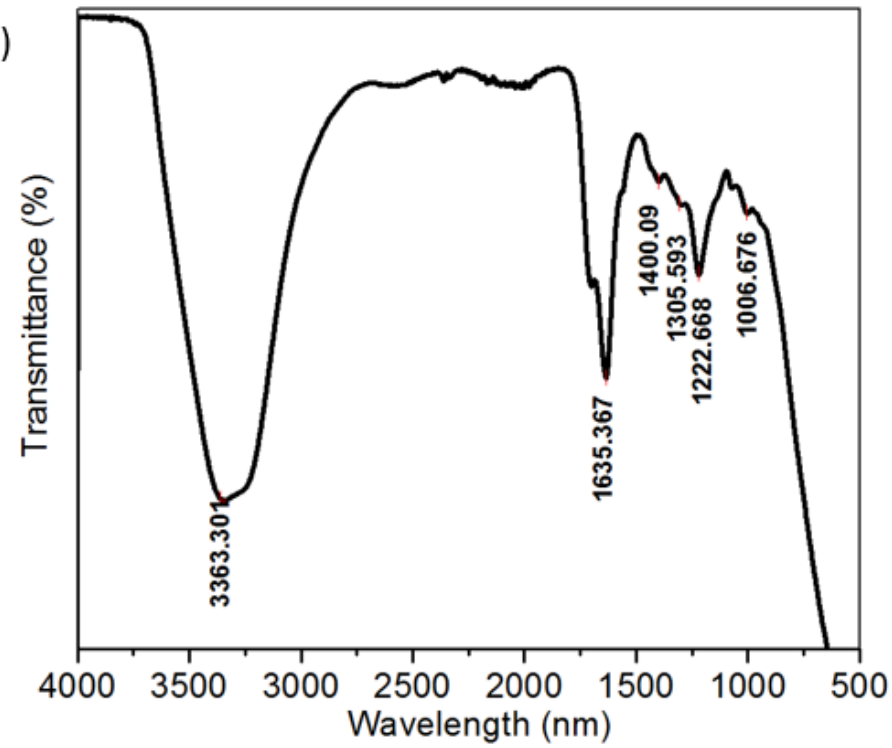

Figure 24. FTIR spectrum of the CNPs solution 
The Fourier transform infrared (FTIR) spectra is further employed to determine the functional groups of the CNPs and the result are shown in Figure 24. The peaks at $1635 \mathrm{~cm}^{-1}$ and $1400 \mathrm{~cm}^{-1}$ are attributed to the $\mathrm{C}=\mathrm{O}$ binding vibration. The peak at 1305 $\mathrm{cm}^{-1}$ and $1222 \mathrm{~cm}^{-1}$ are assigned to the stretching vibration of $\mathrm{C}-\mathrm{N}$ bond. The results here suggest that several functional groups might exist on the surface of the CNPs, including amine, hydroxyl and carboxylic groups, which endow the as-prepared CNPs hydrophilicity and stability in aqueous phase.

\subsubsection{X-ray photoelectron spectrum}

Furthermore, the surface elements and functional groups were also demonstrated by using XPS analysis (Figure 25). The XPS survey spectrum result (Figure 25. a) indicates that the as-synthesized CNPs are comprised of carbon, nitrogen and oxygen elements. The high resolution spectrum of $\mathrm{C} 1 \mathrm{~s}$ exhibits 4 peaks as shown in Figure $25 \mathrm{~b}$ ). The binding peaks at $284.6 \mathrm{eV}, 285.8 \mathrm{eV}, 287.5 \mathrm{eV}$ and $289.2 \mathrm{eV}$ are assigned to the carbon in the form of $\mathrm{C}-\mathrm{C}\left(\mathrm{sp}^{3}\right), \mathrm{C}-\mathrm{N}\left(\mathrm{sp}^{3}\right), \mathrm{C}=\mathrm{O}$ or $\mathrm{C}=\mathrm{N}\left(\mathrm{sp}^{2}\right)$ and the type of $\mathrm{O}-\mathrm{C}=\mathrm{O}$ in polymer, respectively. The high resolution of $\mathrm{N} 1 \mathrm{~s}$ (Figure 25. c) revels the presence of $\mathrm{N}-\mathrm{H}, \mathrm{C}-\mathrm{N}, \mathrm{C}=\mathrm{N}$, which corresponding peak centered at $398.8 \mathrm{eV}, 400.1$ $\mathrm{eV}$ and $402.5 \mathrm{eV}$, respectively. The $\mathrm{O} 1 \mathrm{~s}$ spectrum (Figure 25. d) can be decomposed into peaks at $529.8 \mathrm{eV}, 531.3 \mathrm{eV}$ and $532.9 \mathrm{eV}$, demonstrating the existence of $-\mathrm{OH}$, ${ }^{*} \mathrm{O}=\mathrm{C}-\mathrm{O}$ and $\mathrm{O}=\mathrm{C}-\mathrm{O} *$ 

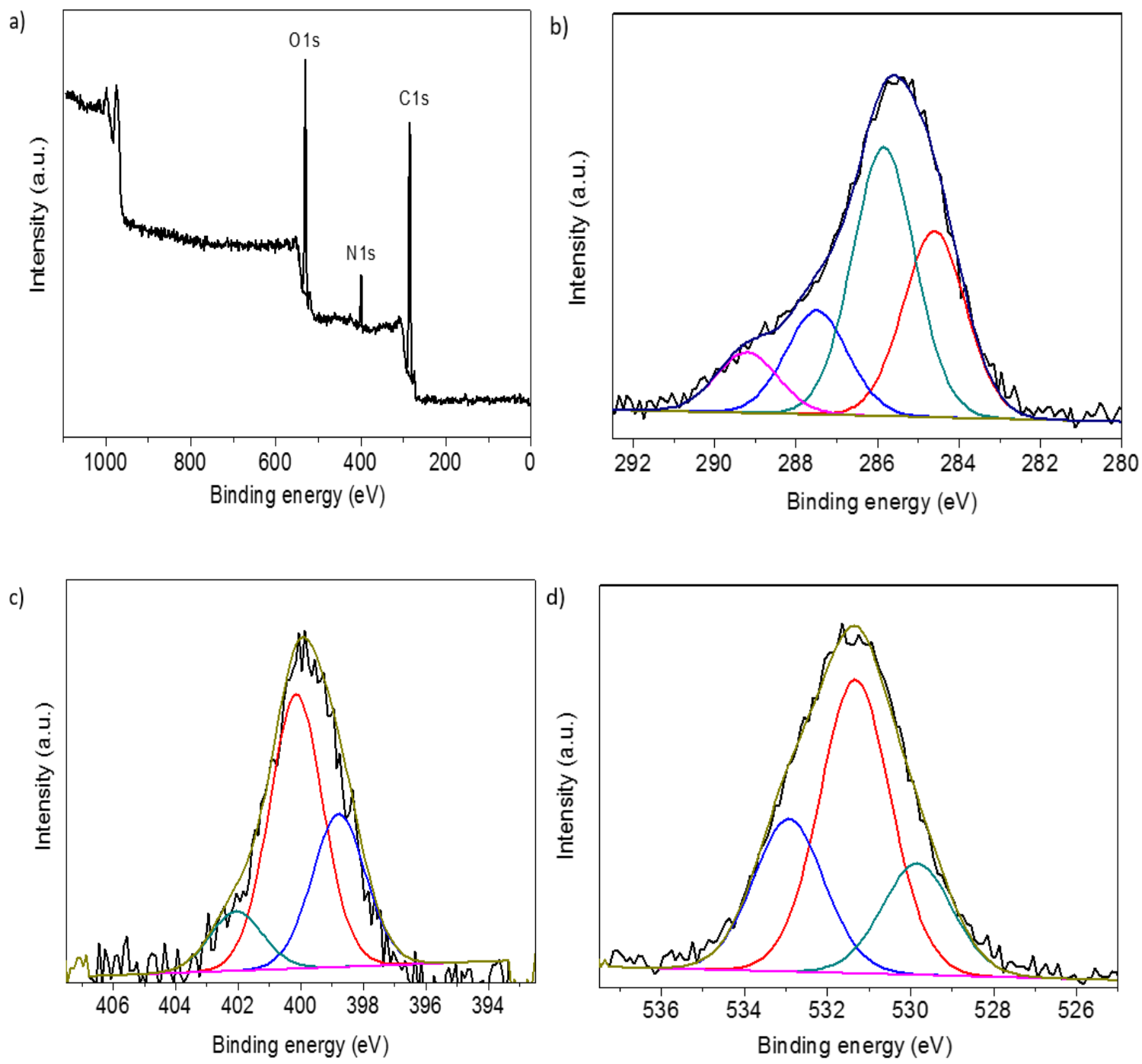

Figure. 25 XPS spectrum of CNPs. a) XPS survey spectrum. b), c) and d) reveal the high resolution XPS spectrum of the $\mathrm{C} 1 \mathrm{~s}, \mathrm{~N} 1 \mathrm{~s}$ and $\mathrm{O} 1 \mathrm{~s}$, respectively. 


\subsection{Optical property of CNPs}

\subsubsection{Excitation and emission spectrum}

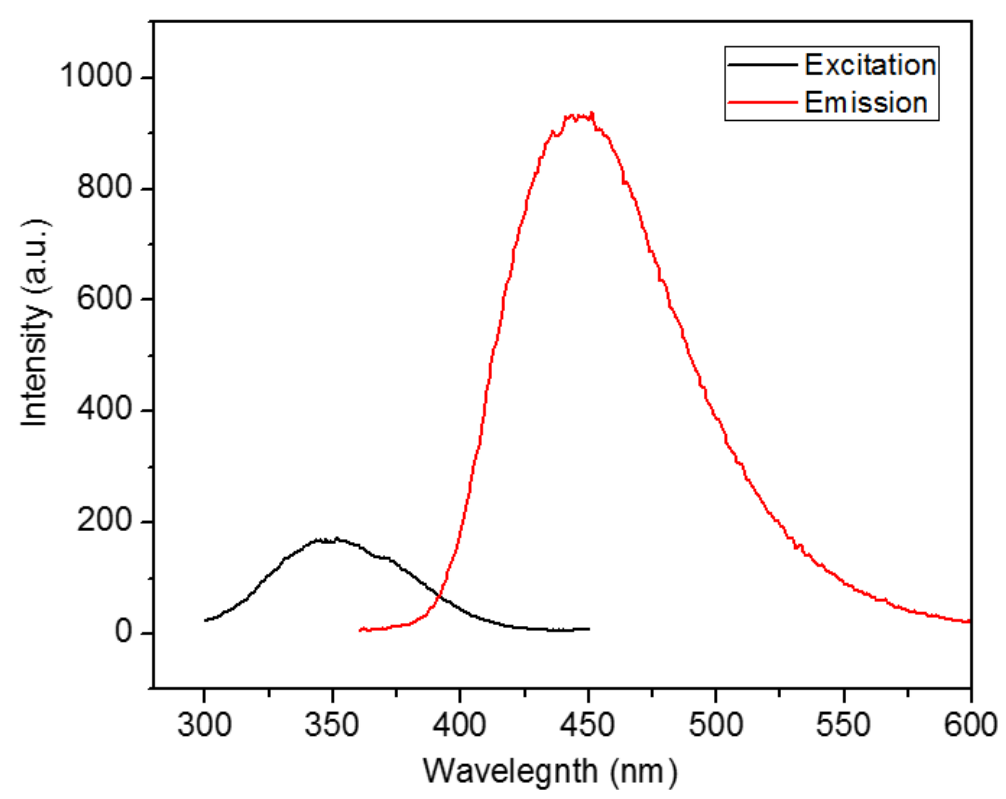

Figure 26. Excitation and emission spectrum of the diluted CNPs solution using $\lambda_{\text {emission }}=450 \mathrm{~nm}$ and $\lambda_{\text {excitation }}=350 \mathrm{~nm}$, respectively.

Figure 26 shows the excitation and emission spectrum of the diluted CNPs solution under the emissim wavelength and excitation wavelength of $450 \mathrm{~nm}$ and 350 $\mathrm{nm}$, respentively. The emission spectrum of carbon nanoparticles with excitation of 350 $\mathrm{nm}$ shows that an emission peak at $450 \mathrm{~nm}$ is observed, indicating the blue emission of carbon nanoparticles. The excitation spectrum (using $\lambda_{\text {emission }}=450 \mathrm{~nm}$ ) displays a broader peak centered at $350 \mathrm{~nm}$, presumably implying one kind of excitation energy trapped on the surface of the carbon nanoparticles. 


\subsubsection{Photographs of the CNPs excited by LEDs}

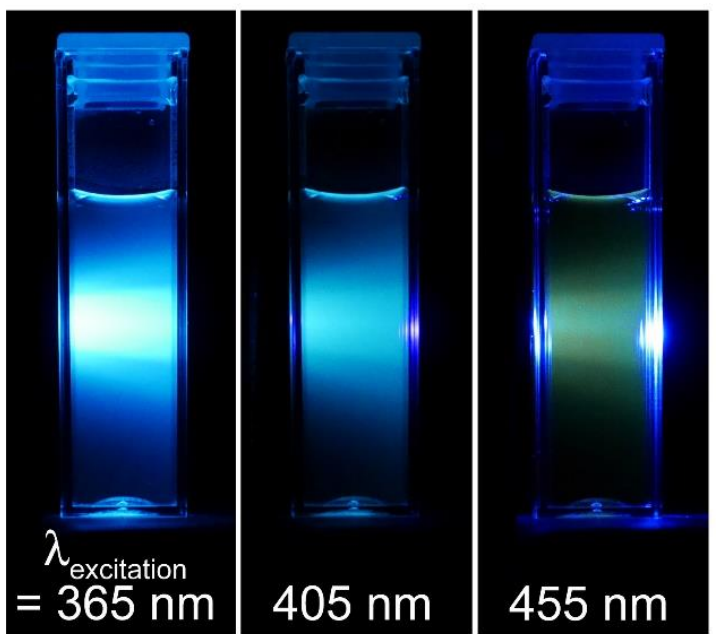

Figure 27. Photographs of the diluted CNPs solution (in water; quartz cuvettes) excitid with fibreoptics-coupled LEDs with different wavelengths indicated in the figure.

The CNPs solution excitated using $365 \mathrm{~nm}$ and $405 \mathrm{~nm}$ wavelength LEDs exhibited blue fluorescence emission PL behavoir of the as-prepared CNPs solution (Figure 6). However, the CNPs solution displays the cyan fluorescence using longer wavelength LEDs (e.g., $420 \mathrm{~nm}$ and $455 \mathrm{~nm}$ ) for excitation due to the brode range of the emission peak (from 400 to $550 \mathrm{~nm}$ ) observed in Figure 26 and 29. 
4.2.3. UV-vis absorption spectrum

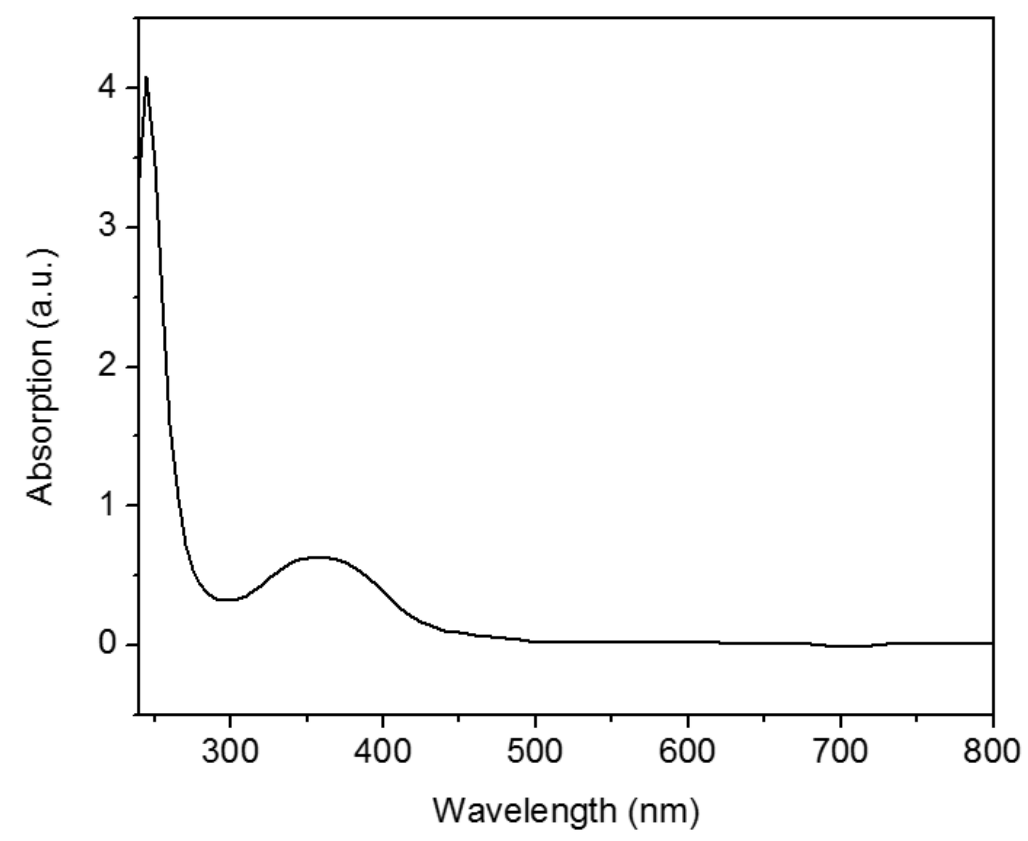

Figure 28. UV-vis absorption spectrum of CNPs in aqueous soulution in the range of $245-800 \mathrm{~nm}$.

The UV-vis spectrum of the diluted CNPs solution in water (Figure 28) exhibits two peaks at $240 \mathrm{~nm}$ and $350 \mathrm{~nm}$, which were ascribed to $\mathrm{n}-\pi^{*}$ transition of $\mathrm{C}=\mathrm{O}$ bond and $\pi-\pi^{*}$ transition of the $\mathrm{C}=\mathrm{C}$ bond, respectively. 


\subsubsection{Emission scan spectrum}

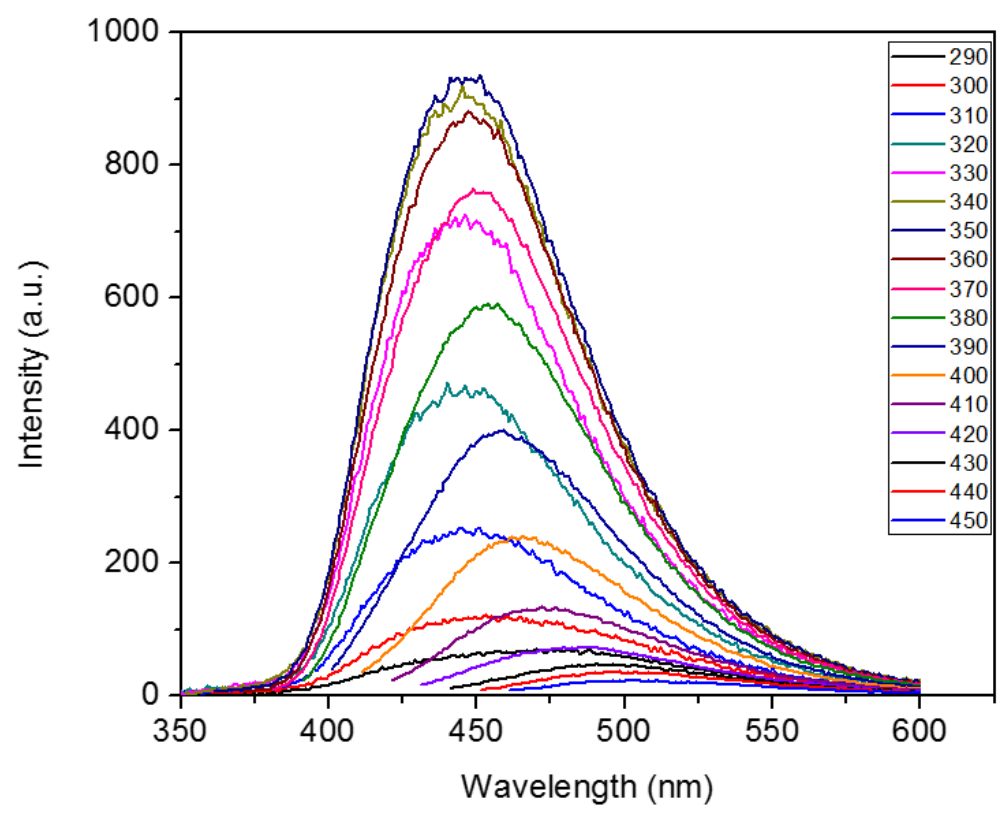

Figure 29. The emission scan spectrum of diluted CNPs with excitation wavelength in the range of $290-450 \mathrm{~nm}$.

Figure 29 illustrates the detailed emission spectra with various excitation wavelengths from 290 to $450 \mathrm{~nm}$ with an increment of $10 \mathrm{~nm}$. When the excitation wavelength is varied from 290 to $390 \mathrm{~nm}$, no obvious shift is observed for the fluorescence emission peak, which centers at $\sim 450 \mathrm{~nm}$ with the highest emission intensity occurred at the excitation wavelength of $350 \mathrm{~nm}$. This result is consisted with the fact that absorption peak is observed at $\sim 350 \mathrm{~nm}$ in Figure 28. Further increasing of excitation wavelength, the corresponding emission peaks shift bathochromically, but the intensities are greatly decreased. This kind of excitation dependent PL behavior is common in fluorescent carbon materials, and is possibly contributed to the surface state affecting the band gap of CNPs or different nanoparticle compositions and structures as well as nanoparticles of different sizes. ${ }^{9,}, 15,43$ 
4.3. Quantitative analysis and selective detection of $\mathrm{Pd}^{2+}$

Owing to the interesting PL behavior and good water solubility/stability, the fluorescent carbon nanoparticles have been widely applied as chemosensors for metal ions detection, in which the functional groups on nanoparticles played a pivotal role. Surface functionalization of the CNPs could be realized through synthesis using different precursors or doping elements. ${ }^{11}$ Especially, the abundant surface functional groups (carboxyl groups, hydroxyl groups and amine groups, etc.) on the surface acted as ligands to interact with metal ions sensitively and selectively. For example, the fluorescence of CNPs was quenched in the presence of metal ions (such as $\mathrm{Fe}^{3+}, \mathrm{Cu}^{2+}$, $\mathrm{Hg}^{2+}$ ) through electron transfer between the CNPs and metal ions, inner filter effect, and charge transfer. However, to the best of our knowledge, $\mathrm{Pd}^{2+}$ detection through fluorescent CNPs has not been reported.

The study of fluorescence quenching in the presence of $\mathrm{Pd}^{2+}$ was carried out by titration experiments upon addition of various concentration of $\mathrm{Pd}^{2+}$ into diluted CNPs solution. As shown in Figure 30. a), the fluorescence intensity of CNPs with the emission peak center at $\sim 450 \mathrm{~nm}$ gradually decreased with the increasing concentration of $\mathrm{Pd}^{2+}$ and then fully quenched by $8 \mu \mathrm{M}$ of $\mathrm{Pd}^{2+}$ under the excitation wavelength of 350 $\mathrm{nm}$. The detection limit of $12.4 \mathrm{nM}$ was calculated using $3 \sigma /$ slope, while $\sigma$ represents standard deviation. In addition, the intensities for the CNP emission peaks at $450 \mathrm{~nm}$ were plotted versus the concentration of $\mathrm{Pd}^{2+}$ and are presented in Figure 10. The quenching results could be analyzed and fitted with the Stern-Volmer (S-V) equation.

$$
I d /=K s v[C]+1 \quad \text { Equation } 2
$$


Where, $I_{0}$ is the fluorescence intensity of the CNPs before the addition of $\mathrm{Pd}^{2+}$ solutions, I is the fluorescence intensity of the CNPs with $\mathrm{Pd}^{2+}, \mathrm{Ksv}$ is the quenching constant and $\mathrm{C}$ is the corresponding molar concentrations of $\mathrm{Pd}^{2+}$. The $\mathrm{PL}$ quenching data showed an exponential S-V curve with the increasing concentration of $\mathrm{Pd}^{2+}(0-8$ $\mu M)$. From the inset of Figure 30. b), a good linear correlation $\left(R^{2}=0.994\right)$ was observed in the presence of lower $\mathrm{Pd}^{2+}$ concentration range $(0-3 \mu \mathrm{M})$. The fluorescent carbon nanoparticles show high sensitivity to $\mathrm{Pd}^{2+}$ detection, which could be attributed to the strong complexing interaction between $\mathrm{Pd}^{2+}$ and surface functional groups of CNPs (e.g., $-\mathrm{NH}_{2}$ ), which results in a nonradiative electron transfer from the excited state of the CNPs to $\mathrm{Pd}^{2+}$. In addition, $\mathrm{Pd}^{2+}$ possesses a square-planar structure, which might allow its easy access and insertion into the surface function group layer of CNPs, resulting in the close contact of $\mathrm{Pd}^{2+}$ with CNPs for electron transfer.
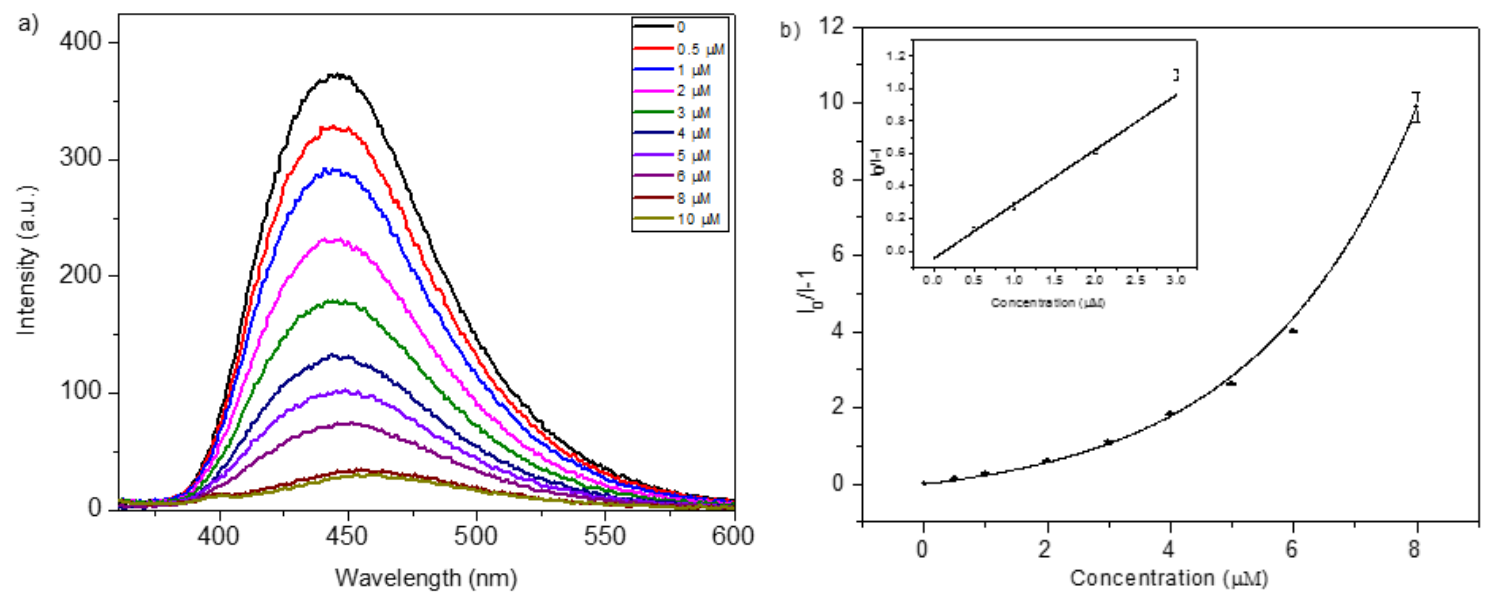

Figure 30. a) PL spectra of the diluted CNPs upon addition of different $\mathrm{Pd}^{2+}(0-10 \mu \mathrm{M})$ concentrations; b) Fitting curve of the nonlinear Stern-Volmer plot for $\mathrm{Pd}^{2+}$ by exponential quenching equation, inset shows the fitting curve of the linearity of the Stern-Volmer plot for $\mathrm{Pd}^{2+}$ of $0-3 \mu \mathrm{M}$.

In order to evaluate the selectivity of the as-prepared CNPs toward $\mathrm{Pd}^{2+}$ detection, fluorescence quenching titrations were also performed using other competing metal ions. The fluorescence quenching efficiencies of 13 kinds of metal ions at $50 \mu \mathrm{M}$ 
are shown in Figure 31. Except for $\mathrm{Hg}^{2+}$ and $\mathrm{Fe}^{3+}$, they caused fluorescence quenching by about $80 \%$ and $50 \%$ at $50 \mu \mathrm{M}$ level (which is still not significant considering the fully quenching of CNPs by $\mathrm{Pd}^{2+}$ at $8 \mu \mathrm{M}$ ), other metal ions did not obviously change the fluorescence intensity of CNPs solution. Although citric acid and PEI have been used to prepare carbon dots using microwave for sensitive and selective detection $\mathrm{Cu}^{2+42}$, our data show that the quenching for $\mathrm{Pd}^{2+}$ is more premium than that for $\mathrm{Cu}^{2+}$, which might be attributed to the different size and surface functional groups, resulted from the different microwave reaction conditions (much lower watts with much longer microwave time in this study vs. much higher watts with much shorter time in other report). The XPS data in Figure 25 successfully demonstrated the presence of various functional groups on the surface of the as-prepared CNPs, however, detailed comparison between the CNPs prepared in this study and the one reported in literature for $\mathrm{Cu}^{2+}$ sensing ${ }^{42}$ is impossible as there is no such information available in the other report. These results revealed that the CNPs-based sensor possesses high potential for sensitive and selective detection of $\mathrm{Pd}^{2+}$.In order to determine the selectivity of the as-prepared CNPs toward $\mathrm{Pd}^{2+}$, the CNPs titrated by other competing metal ions were tested. Figure 31 represented the fluorescence quenching efficiency of 13 kinds of metal ions (50 $\mu \mathrm{M})$. Except $\mathrm{Hg}^{2+}$ and $\mathrm{Fe}^{3+}$ decreased the $80 \%$ and $50 \%$ initial PL intensity, other metal ions did not obviously change the fluorescence intensity compared with that was fully quenched by $\mathrm{Pd}^{2+}$. Furthermore, the fluorescence quenching phenomenon was also observed with the naked eyes under the UV lamp $(\lambda e x c i t a t i o n=365 \mathrm{~nm})$ as shown in Figure 32. These results revealed that the CNPs-based sensor exhibited good capability to detect $\mathrm{Pd}^{2+}$ sensitivity and selectivity. 


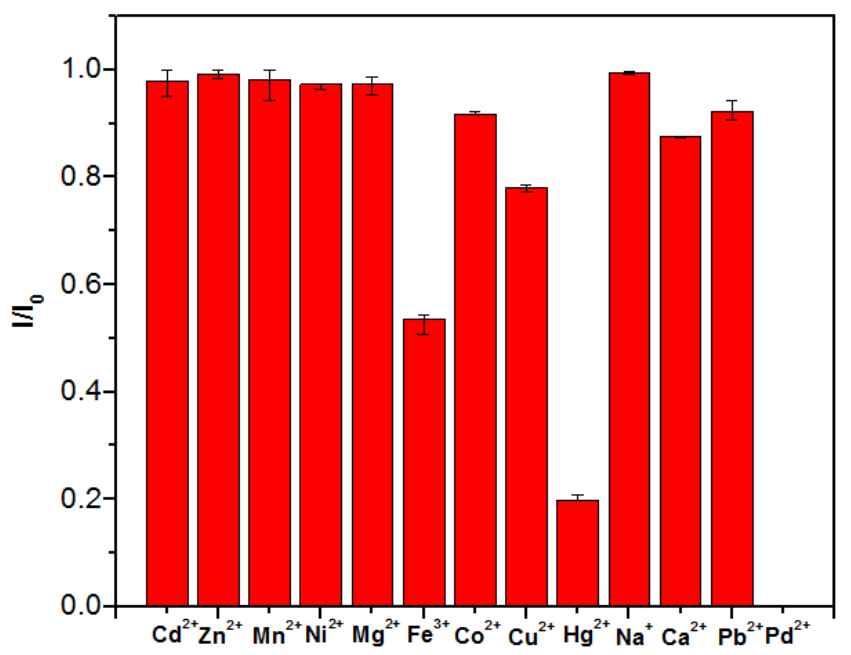

Figure. 31 Fluorescence quenching efficiency obtained for CNPs solution with $\mathrm{Pd}^{2+}(10 \mu \mathrm{M})$ and 12 other competing metal ions at $50 \mu \mathrm{M}$, indicating good selectivity for $\mathrm{Pd}^{2+}$ sensing.
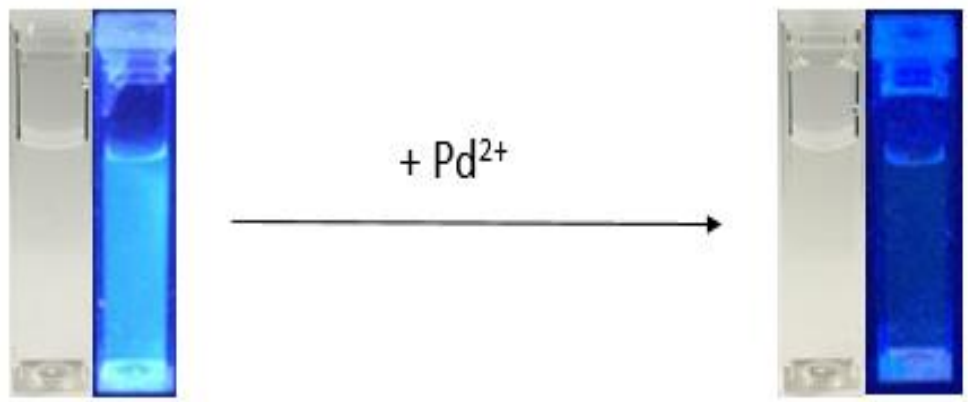

Figure. 32, the pictures of diluted CNPs in aqueous solution under white light and UV lamp $(\lambda$ excitation $=365 \mathrm{~nm})$ in the absence and presence of $\mathrm{Pd} 2+(50 \mu \mathrm{M})$. 


\section{Conclusion}

In summary, we have synthesized fluorescent nanoparticles by using citric acid and PEI as precursors with microwave-assisted method. The formation of the nitrogendoped carbon nanoparticles was demonstrated by various characteristic methods. The as-synthesized showed good solubility and stability in aqueous solution and excitationdependent PL property. The no further modification needed carbon nanoparticles also displayed high sensitivity toward $\mathrm{Pd}^{2+}$ that the limit of detection could reach as low as $12.4 \mathrm{nM}$. The selectivity of the carbon nanoparticles for $\mathrm{Pd}^{2+}$ sensing was demonstrated by fluorescence quenching titration with a number of competing metal ions. These findings suggest that the as-prepared carbon nanoparticles provide a new strategy for

$\mathrm{Pd}^{2+}$ sensing, which possessed the advantages of simplicity, cost-effectiveness and environment friendly compared with conventional methods with organic dyes. 


\section{Outlook}

Although various applications have been exploited using CNPs, including bioimaging, nanoplatform therapy, heavy metal ions detection, fluorescent ink, explosive detection and light harvesting, its wider application as a class of fluorescent materials was greatly hurdled compared to other common fluorescent molecules, due to the limit information and understanding of fundamentals in materials structure-propertyfunction relationship. The preparation of CNPs with unique fluorescence property and its applications in the past is basically on a basis of trial-and-error, which is lack of systematic study and cannot generate a full picture. Therefore, there are two overall future research trends in developing unique CNPs and exploiting its applications: 1) increased efforts in fundamental studies for better understanding of the relation between CNPs structure and the optical properties. Most of the CNPs have so far been developed empirically. The structure and properties of CNPs should be characterized carefully and modeling and simulation can be involved to establish guidelines for the design of CNPs and develop reliable criteria for predicting the most useful CNPs for individual applications; and 2) New or improved CNPs synthesis with full controllability of CNPs properties is required. Currently many optical and electronic properties of CNPs have not been well controlled because of the unpredictable synthesis process of CNPs, resulting in the unpredictable chemical structure of CNPs. Although CNPs synthesis using inexpensive, recycling source and easy obtained natural materials, such as food waste ${ }^{44}$, milk ${ }^{34}$, cotton $^{29}$ and soy milk ${ }^{45}$ as precursors have gained much attention as green synthetic route in recent years, the complicity of the precursors could cause more obstacles in 
understanding the relationship between material structure and material properties. In this regard, materials simulation is a very powerful tool to understand the correlation of material structure and properties, however, it barely employed in the design/selection of CNPs up to date. With the establishment of more and more accurate materials models to reveal the structure-property-function relationship, a computation method to project the application of various functional materials or composites is expected to play a critical role in future research of CNPs.

We believe that with further research efforts in CNPs, functional CNPs with improved and controllable optical properties will endow CNPs unprecedented power in various applications and also serve as an alternative for traditional organic fluorescent dye and inorganic semiconductor quantum dots. 


\section{References}

1. Kairdolf, B.A., et al., Semiconductor quantum dots for bioimaging and biodiagnostic applications. Annual review of analytical chemistry (Palo Alto, Calif.), 2013. 6(1): p. 143.

2. $\mathrm{Xu}, \mathrm{X}$., et al., Electrophoretic analysis and purification of fluorescent single-walled carbon nanotube fragments. Journal of the American Chemical Society, 2004. 126(40): p. 12736-12737.

3. Shi, L., et al., Naked oats-derived dual-emission carbon nanodots for ratiometric sensing and cellular imaging. Sensors and Actuators B: Chemical, 2015. 210: p. 533-541.

4. Gao, Z., et al., A carbon dot-based "off-on" fluorescent probe for highly selective and sensitive detection of phytic acid. Biosensors and Bioelectronics, 2015. 70: p. 232-238.

5. Yu, C., et al., Carbon-dot-based ratiometric fluorescent sensor for detecting hydrogen sulfide in aqueous media and inside live cells. Chemical Communications, 2013. 49(4): p. 403-405.

6. Ge, J., et al., Carbon Dots with Intrinsic Theranostic Properties for Bioimaging, Red - Light - Triggered Photodynamic/Photothermal Simultaneous Therapy In Vitro and In Vivo. Advanced healthcare materials, 2015.

7. Jiang, Y., et al., A fluorescence turn-off chemosensor based on $\mathrm{N}$-doped carbon quantum dots for detection of $\mathrm{Fe} 3+$ in aqueous solution. Materials Letters, 2015. 141: p. 366-368.

8. Wang, C., et al., Simple and green synthesis of nitrogen-, sulfur-, and phosphorus-co-doped carbon dots with tunable luminescence properties and sensing application. RSC Advances, 2014. 4(96): p. 5406054065.

9. Sun, X., C. Brückner, and Y. Lei, One-pot and ultrafast synthesis of nitrogen and phosphorus co-doped carbon dots possessing bright dual wavelength fluorescence emission. Nanoscale, 2015. 7(41): p. 1727817282.

10. Wang, Y., et al., FRET-and PET-based sensing in a single material: expanding the dynamic range of an ultra-sensitive nitroaromatic explosives assay. Chemical Communications, 2012. 48(79): p. 9903-9905.

11. Wang, C., et al., Upconversion fluorescent carbon nanodots enriched with nitrogen for light harvesting. Journal of Materials Chemistry, 2012. 22(31): p. 15522-15525.

12. Cui, Y., et al., Simple and Efficient Synthesis of Strongly Green Fluorescent Carbon Dots with Upconversion Property for Direct Cell Imaging. Particle \& Particle Systems Characterization, 2015. 32(5): p. 542-546.

13. Liu, S., et al., A general strategy for the production of photoluminescent carbon nitride dots from organic amines and their application as novel peroxidase-like catalysts for colorimetric detection of $\mathrm{H} 2 \mathrm{O} 2$ and glucose. RSC Advances, 2012. 2(2): p. 411-413.

14. Peng, H. and J. Travas-Sejdic, Simple aqueous solution route to luminescent carbogenic dots from carbohydrates. Chemistry of Materials, 2009. 21(23): p. 5563-5565.

15. Qu, S., et al., A Biocompatible Fluorescent Ink Based on Water - Soluble Luminescent Carbon Nanodots. Angewandte Chemie, 2012. 124(49): p. 12381-12384.

16. Xiangcheng Sun, J.H., Yongtao Meng, Lichun Zhang, Sichen Zhang, Xiaoyu Ma, Swayandipta Dey, Jing Zhao and Yu Lei, Microwave-assisted ultrafast and facile synthesis of fluorescent carbon nanoparticles from single precursor: Preparation, characterization and its application for highly selective detection of explosive picric acid Journal of Materials Chemistry A, 2015.

17. Pan, D., et al., Observation of $\mathrm{pH}$-, solvent-, spin-, and excitation-dependent blue photoluminescence from carbon nanoparticles. Chemical Communications, 2010. 46(21): p. 3681-3683.

18. Zhou, L., et al., Carbon nanodots as fluorescence probes for rapid, sensitive, and label-free detection of $\mathrm{Hg}$ $2+$ and biothiols in complex matrices. Chemical communications, 2012. 48(8): p. 1147-1149.

19. Hu, S.-L., et al., One-step synthesis of fluorescent carbon nanoparticles by laser irradiation. Journal of Materials Chemistry, 2009. 19(4): p. 484-488.

20. Liu, H., T. Ye, and C. Mao, Fluorescent carbon nanoparticles derived from candle soot. Angewandte Chemie International Edition, 2007. 46(34): p. 6473-6475.

21. Gong, X., et al., Facile synthesis of nitrogen-doped carbon dots for Fe 3+ sensing and cellular imaging. Analytica chimica acta, 2015. 861: p. 74-84.

22. Jiang, K., et al., Bright-Yellow-Emissive N-Doped Carbon Dots: Preparation, Cellular Imaging, and Bifunctional Sensing. ACS applied materials \& interfaces, 2015. 7(41): p. 23231-23238. 
23. Lan, M., et al., A recyclable carbon nanoparticle-based fluorescent probe for highly selective and sensitive detection of mercapto biomolecules. Journal of Materials Chemistry B, 2015. 3(1): p. 127-134.

24. Wang, W., et al., Facile synthesis of N, S-codoped fluorescent carbon nanodots for fluorescent resonance energy transfer recognition of methotrexate with high sensitivity and selectivity. Biosensors and Bioelectronics, 2015. 64: p. 517-522.

25. Guo, Y., et al., Fluorescent carbon nanoparticles for the fluorescent detection of metal ions. Biosensors and Bioelectronics, 2015. 63: p. 61-71.

26. Guo, Y., et al., Hydrothermal synthesis of highly fluorescent carbon nanoparticles from sodium citrate and their use for the detection of mercury ions. Carbon, 2013. 52: p. 583-589.

27. Dong, Y., et al., Polyamine-functionalized carbon quantum dots as fluorescent probes for selective and sensitive detection of copper ions. Analytical chemistry, 2012. 84(14): p. 6220-6224.

28. Cao, B., et al., Ratiometric fluorescence detection of mercuric ion based on the nanohybrid of fluorescence carbon dots and quantum dots. Analytica chimica acta, 2013. 786: p. 146-152.

29. Wen, X., et al., Green synthesis of carbon nanodots from cotton for multicolor imaging, patterning, and sensing. Sensors and Actuators B: Chemical, 2015. 221: p. 769-776.

30. Li, H., et al., Carbon nanoparticle for highly sensitive and selective fluorescent detection of mercury (II) ion in aqueous solution. Biosensors and Bioelectronics, 2011. 26(12): p. 4656-4660.

31. Lan, M., et al., Carbon Nanoparticle-based Ratiometric Fluorescent Sensor for Detecting Mercury lons in Aqueous Media and Living Cells. ACS applied materials \& interfaces, 2014. 6(23): p. 21270-21278.

32. Liu, S., et al., Hydrothermal Treatment of Grass: A Low - Cost, Green Route to Nitrogen - Doped, Carbon Rich, Photoluminescent Polymer Nanodots as an Effective Fluorescent Sensing Platform for Label - Free Detection of Cu (II) lons. Advanced Materials, 2012. 24(15): p. 2037-2041.

33. Qu, F., et al., Differentiation of multi-metal ions based on fluorescent dual-emission carbon nanodots. RSC Advances, 2015. 5(100): p. 82570-82575.

34. Wang, J., et al., Large - Scale Green Synthesis of Fluorescent Carbon Nanodots and Their Use in Optics Applications. Advanced Optical Materials, 2015. 3(1): p. 103-111.

35. Sadhanala, H.K. and K.K. Nanda, Boron and Nitrogen Co-Doped Carbon Nanoparticles as Photoluminescent Probes for Selective and Sensitive Detection of Picric Acid. The Journal of Physical Chemistry C, 2015.

36. Duan, L., Y. Xu, and X. Qian, Highly sensitive and selective Pd2+ sensor of naphthalimide derivative based on complexation with alkynes and thio-heterocycle. Chem. Commun., 2008(47): p. 6339-6341.

37. Balamurugan, R., et al., A depropargylation-triggered fluorescence "turn-on" probe for the detection of $P d$ 2+ based on a bispropargylamine-rhodamine conjugate. Analyst, 2013. 138(5): p. 1564-1569.

38. $\mathrm{Li}, \mathrm{H}$. , et al., A fluorescent and colorimetric probe specific for palladium detection. Chemical Communications, 2010. 46(7): p. 1079-1081.

39. Santra, M., et al., Fluorescent detection of palladium species with an O-propargylated fluorescein. Chemical Communications, 2010. 46(22): p. 3964-3966.

40. Sun, X., Y. Wang, and Y. Lei, Fluorescence based explosive detection: from mechanisms to sensory materials. Chemical Society Reviews, 2015. 44(22): p. 8019-8061.

41. Wang, M., et al., Highly Selective and Reversible Chemosensor for Pd2+ Detected by Fluorescence, Colorimetry, and Test Paper. ACS applied materials \& interfaces, 2015. 7(2): p. 1284-1289.

42. Salinas-Castillo, A., et al., Carbon dots for copper detection with down and upconversion fluorescent properties as excitation sources. Chemical Communications, 2013. 49(11): p. 1103-1105.

43. Zhu, S., et al., Highly photoluminescent carbon dots for multicolor patterning, sensors, and bioimaging. Angewandte Chemie, 2013. 125(14): p. 4045-4049.

44. Park, S.Y., et al., Photoluminescent green carbon nanodots from food-waste-derived sources: Large-scale synthesis, properties, and biomedical applications. ACS applied materials \& interfaces, 2014. 6(5): p. 33653370.

45. Zhu, C., J. Zhai, and S. Dong, Bifunctional fluorescent carbon nanodots: green synthesis via soy milk and application as metal-free electrocatalysts for oxygen reduction. Chemical Communications, 2012. 48(75): p. 9367-9369. 